\title{
MEL-18 loss mediates estrogen receptor- $\alpha$ downregulation and hormone independence
}

\author{
Jeong-Yeon Lee, ${ }^{1}$ Hee-Young Won, ${ }^{2}$ Ji-Hye Park, ${ }^{1}$ Hye-Yeon Kim, ${ }^{2}$ Hee-Joo Choi, ${ }^{2}$ Dong-Hui Shin, ${ }^{2}$ Ju-Hee Kang, ${ }^{3}$ \\ Jong-Kyu Woo, ${ }^{4}$ Seung-Hyun Oh, ${ }^{4}$ Taekwon Son, ${ }^{5}$ Jin-Woo Choi, ${ }^{2}$ Sehwan Kim, ${ }^{6}$ Hyung-Yong Kim, ${ }^{2,6}$ Kijong Yi, ${ }^{2}$ \\ Ki-Seok Jang, ${ }^{2}$ Young-Ha Oh, ${ }^{2}$ and Gu Kong ${ }^{1,2}$ \\ IInstitute for Bioengineering and Biopharmaceutical Research (IBBR) and ${ }^{2}$ Department of Pathology, College of Medicine, Hanyang University, Seoul, South Korea. ${ }^{3}$ National Cancer Center, \\ Goyang-si, Gyeonggi-do, South Korea. “'College of Pharmacy, Gachon University, Incheon, South Korea. ${ }^{5}$ Research Institute, Bio-Medical Science Co., Ltd., Daejeon, South Korea. \\ ${ }^{6}$ Data Science Center, Insilicogen Inc., Suwon-si, Gyeonggi-do, South Korea.
}

\begin{abstract}
The polycomb protein MEL-18 has been proposed as a tumor suppressor in breast cancer; however, its functional relevance to the hormonal regulation of breast cancer remains unknown. Here, we demonstrated that MEL-18 loss contributes to the hormone-independent phenotype of breast cancer by modulating hormone receptor expression. In multiple breast cancer cohorts, MEL-18 was markedly downregulated in triple-negative breast cancer (TNBC). MEL-18 expression positively correlated with the expression of luminal markers, including estrogen receptor- $\alpha$ (ER- $\alpha$, encoded by ESR1). MEL-18 loss was also associated with poor response to antihormonal therapy in ER- $\alpha$-positive breast cancer. Furthermore, whereas MEL-18 loss in luminal breast cancer cells resulted in the downregulation of expression and activity of ER- $\alpha$ and the progesterone receptor (PR), MEL-18 overexpression restored ER- $\alpha$ expression in TNBC. Consistently, in vivo xenograft experiments demonstrated that MEL-18 loss induces estrogen-independent growth and tamoxifen resistance in luminal breast cancer, and that MEL-18 overexpression confers tamoxifen sensitivity in TNBC. MEL-18 suppressed SUMOylation of the ESR1 transactivators p53 and SP1, thereby driving ESR1 transcription. MEL-18 facilitated the deSUMOylation process by inhibiting BMI-1/RINC1B-mediated ubiquitin-proteasomal degradation of SUM01/sentrin-specific protease 1 (SENP1). These findings demonstrate that MEL-18 is a SUMO-dependent regulator of hormone receptors and suggest MEL-18 expression as a marker for determining the antihormonal therapy response in patients with breast cancer.
\end{abstract}

\section{Introduction}

Estrogen receptor- $\alpha$ (ER- $\alpha)$, a hormone-dependent nuclear receptor encoded by ESR1, is the most common clinical marker used for breast cancer prognosis and the classification of breast cancer subtypes. Approximately $30 \%$ of breast cancers do not express ER- $\alpha$, which is associated with an aggressive phenotype, resistance to antiestrogen therapy, and poor prognosis (1). In these ER- $\alpha$-negative cells, triple-negative breast cancer (TNBC) lacking ER- $\alpha$ and progesterone receptor (PR, encoded by $P G R$ ) expression and epidermal growth factor receptor 2 (HER2) overexpression has no benefits from conventional hormonal and HER2-based therapies, and the molecular mechanism underlying TNBC remains poorly understood. Because the ESR1 expression can be reversibly modulated by several epigenetic factors, including histone modification and DNA methylation (2-4), ER- $\alpha$ re-expression using demethylating or deacetylating agents has been performed to restore the sensitivity of ER- $\alpha$-negative breast cancer and TNBC to endocrine therapy (5). However, the mechanisms underlying the loss of ER- $\alpha$ in breast cancer and the regulation of ESR1 gene transcription are not completely understood.

Conflict of interest: The authors have declared that no conflict of interest exists. Submitted: October 14, 2013; Accepted: February 12, 2015

Reference information: J Clin Invest. 2015;125(5):1801-1814. doi:10.1172/JCI73743.
MEL-18 is a component of polycomb repressive complex-1 (PRC-1), which is a critical epigenetic modulator of stem cell regulation, and normal and cancerous cell development (6). Although further clinical evidence is needed, accumulating studies have suggested that MEL-18 acts as a tumor suppressor in several human tumors, including breast cancer (7-11). Our previous studies have also revealed that MEL-18 loss facilitates stem cell activity, cell growth, angiogenesis, and epithelial-mesenchymal transition (EMT) in breast cancer (12-15), implying an association of MEL-18 loss with an aggressive phenotype. MEL-18 is involved in multiple posttranslational modifications of target proteins and in epigenetic gene regulation. PRC-1, which contains the ubiquitin E3 ligase RING1B, modulates both histone H2A ubiquitination-mediated gene silencing and ubiquitin-proteasome-mediated protein degradation $(16,17)$. We have demonstrated that MEL-18 negatively regulates RING1B activity by inhibiting BMI-1 transcription (18). MEL-18 also acts as an inhibitor of SUMOylation $(19,20)$. However, the importance of MEL-18 in SUMOylation remains unclear.

In this study, we demonstrated that MEL-18 loss is associated with hormone receptor-independent phenotypes, including tamoxifen resistance and TNBC, by regulating SUMOylation/ deSUMOylation-dependent ESR1 and PGR expression, suggesting that MEL-18 may serve as a prognostic indicator of the outcome of antihormonal therapy in breast cancer. 


\section{Results}

The loss of MEL-18 indicates poor prognosis and triple-negativity of human breast cancer. To identify the tumor-suppressive functions of MEL-18 in breast cancer, we first investigated the clinical relevance of MEL-18 in 223 human primary breast cancers. Low MEL-18 expression correlated with larger tumor size $(P=0.001)$ and higher American Joint Committee on Cancer (AJCC) stage $(P=0.021)$ (Supplemental Table 1; supplemental material available online with this article; doi:10.1172/JCI73743DS1). Notably, MEL18 expression was significantly associated with TNBC $(P=0.003)$ among breast cancer subtypes (Figure $1 \mathrm{~A}$ and Supplemental Table 1). In particular, MEL-18 expression strongly correlated with ER- $\alpha$ and PR expression ( $P=0.001$ and 0.029 , respectively) but not HER2 overexpression ( $P=0.164)$ (Figure 1B and Supplemental Table 1). To further validate these results in a broad range of clinical samples, we analyzed several publicly available gene expression microarray datasets of breast cancer patients (21-27). Consistent with our clinical cohort results, $M E L-18$ mRNA levels were significantly lower in TNBC cases than luminal and HER2-positive cases in these independent datasets (Figure 1C). Moreover, correlation analysis revealed that $M E L-18$ expression tended to be positively associated with the expression of the luminal markers ESR1, PGR, GATA3, and FOXA1 (Figure 1D and Table 1).

By analyzing the association of MEL-18 expression with breast cancer patient survival using the Kaplan-Meier method followed by the log-rank test and the Cox regression model, we further confirmed that the loss of MEL-18 correlates with poorer overall survival (OS; $P=0.001$ and 0.003 , respectively) and disease-free survival (DFS; $P=0.011$ and 0.003, respectively, Figure $1 \mathrm{E}$ and Table 2). In addition, multivariate survival analyses revealed that MEL-18 loss is an independent prognostic factor of poor OS (Table 2). TNBC patients who displayed MEL-18 expression also exhibited a tendency toward a more favorable survival outcome than those who displayed MEL-18 negativity, although this difference was not significant (OS, $P=0.083$; DFS, $P=0.178$ ) (Figure $1 \mathrm{E}$ ). Based on a meta analysis-based Kaplan-Meier Plotter containing survival information for 4,142 breast cancer patients (28), we also confirmed a significant association between lower MEL-18 mRNA levels and unfavorable survival, particularly in luminal subtypes (Supplemental Figure 1A and B). However, in basal subtypes, which may already display $M E L$ 18 loss, no significant survival difference was observed between the groups expressing high and low levels of MEL-18 (Supplemental Figure 1C). Taken together, these data suggest that MEL-18 serves as a prognostic factor and a marker of human breast cancer that has a loss associated with hormone receptor negativity and triple negativity.

$M E L-18$ regulates hormone receptors. We also confirmed that MEL-18 was strongly expressed in hormone receptor-positive cancer cell lines, whereas its expression was weak or absent in ER- $\alpha-$ negative and TNBC cell lines (Supplemental Figure 2A). The expression of other polycomb group (PcG) proteins, EZH2 and BMI-1, was not correlated with hormone receptor status in these cell lines. To explore the functional role of MEL-18 in hormone receptor-positive cells, a gene expression microarray was conducted after MEL18 knockdown via shRNA transduction into MCF-7 luminal breast cancer cells. The depletion of MEL-18 induced the downregulation of several luminal markers, including ESR1 and PGR (1.6- and 1.9fold decrease, respectively), but induced the upregulation of various basal markers, including $C A V 1, C A V 2$, and $K R T 17$, compared with the control (Figure 2A). Moreover, Gene Ontology (GO) analysis implied that MEL-18 target genes are highly enriched in hormone-related biological processes, including responses to hormone stimulus and mammary gland development (Figure 2B). Because ER- $\alpha$ and PR are the most prominent factors involved in the hormonal regulation of breast cancer, we further examined the effect of MEL-18 on these hormone receptors. Consistent with the results of the gene expression array, decreased protein and mRNA levels of ER- $\alpha$ and PR were observed following MEL-18 knockdown in two HER2-nonamplified luminal breast cancer cell lines, MCF-7 and T47D (Figure 2, C and D, and Supplemental Figure 2B). Furthermore, MEL-18 overexpression induced the expression of both ESR1 and PGR in MDA-MB-231 and MDA-MB-468 TNBC cells (Figure 2E and Supplemental Figure $2 \mathrm{~B}$ ). In particular, in the TNBC cells, ER- $\alpha$ protein levels were slightly restored by MEL-18 overexpression (Figure 2C). No effect on HER2 expression was observed in either MEL-18-silenced luminal cells or MEL-18-overexpressing TNBC cells. Based on the luciferase reporter assay using previously characterized ESR1 promoters (29), we further confirmed that MEL-18 modulates the activity of a proximal ESR1 promoter, termed ESR1 proAB (Supplemental Figure 2, $\mathrm{C}$ and D). MEL-18 knockdown also decreased PGR promoter activity (Supplemental Figure 2E). Taken together, these data indicate that MEL-18 transcriptionally regulates the expression of hormone receptors in both luminal breast cancer and TNBC cells.

Loss of MEL-18 confers estrogen independence and resistance to antihormonal therapy. We also explored whether the regulation of ER- $\alpha$ expression by MEL-18 affects ER- $\alpha$-dependent transcriptional activity. MEL-18 knockdown abrogated the luciferase activity of $17 \beta$-estradiol-induced (E2-induced) estrogen-response elements (EREs) and the expression of the ER- $\alpha$ target genes TFF1 and PGR in luminal breast cancer cell lines, including MCF-7; alternatively, MEL-18 overexpression restored E2-mediated ERE activity and target gene expression in the TNBC cell lines (Figure 3, A-C, and Supplemental Figure 3, A and B). In addition, decreased PR expression due to MEL-18 knockdown affected PRE luciferase activity (Supplemental Figure 2F). These data indicated that MEL-18 induces hormone receptor activation by modulating their expression. We further investigated the potential feedback mechanism relating MEL-18 expression and hormone activity. However, MEL-18 expression was not altered in response to treatment with estrogen or progesterone (Supplemental Figure 4, A-D). We next examined whether the loss of ER- $\alpha$ expression and activity due to MEL-18 knockdown in hormone receptor-positive cancer cells leads to progression from an estrogen-dependent to an estrogen-independent phenotype. In MCF-7 and T47D cells, MEL-18 loss increased cell growth regardless of E2 treatment (Supplemental Figure 5A). Conversely, the growth retardation caused by MEL-18 overexpression was ameliorated by E2 treatment in MDA-MB-468 and MDA-MB-231 cells, implying the restoration of E2 signaling in TNBC cells due to MEL-18 overexpression. We also confirmed that MEL-18 negatively regulates AKT phosphorylation levels in these cells regardless of E2 status (Supplemental Figure 5B), consistent with our previous report that MEL18 suppresses AKT-dependent breast cancer cell growth (14). We further determined the effect of MEL-18 on estrogen-independent breast tumor growth in vivo. Notably, increased tumor formation was observed in the absence of E2 treatment in mouse xenografts 
A

A Molecular subtypes of breast cancer a Low MEL-18

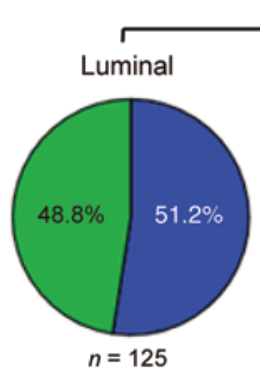

B MEL-18
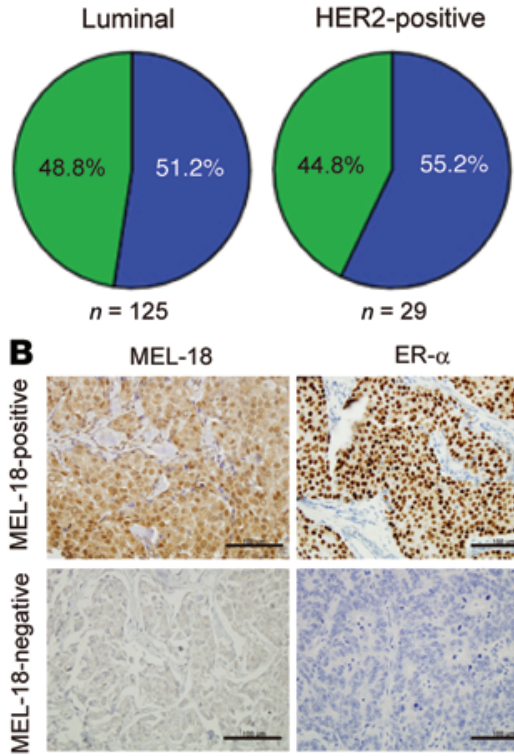

ER- $\alpha$

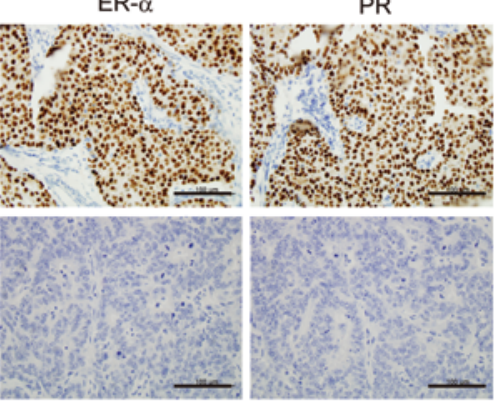

$\square$ MEL-18 positive 口MEL-18 negative
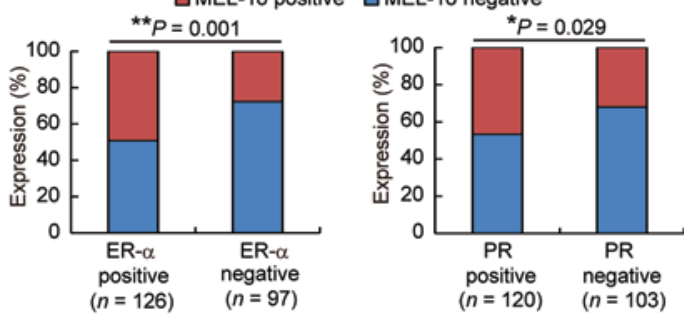

All cases $(n=223)$

E

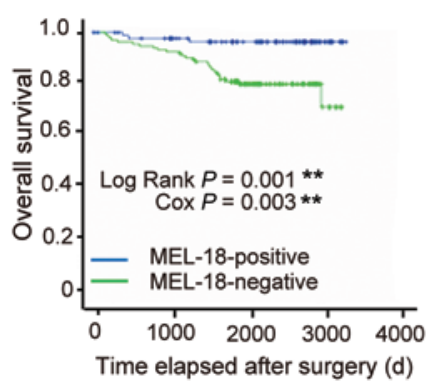

C
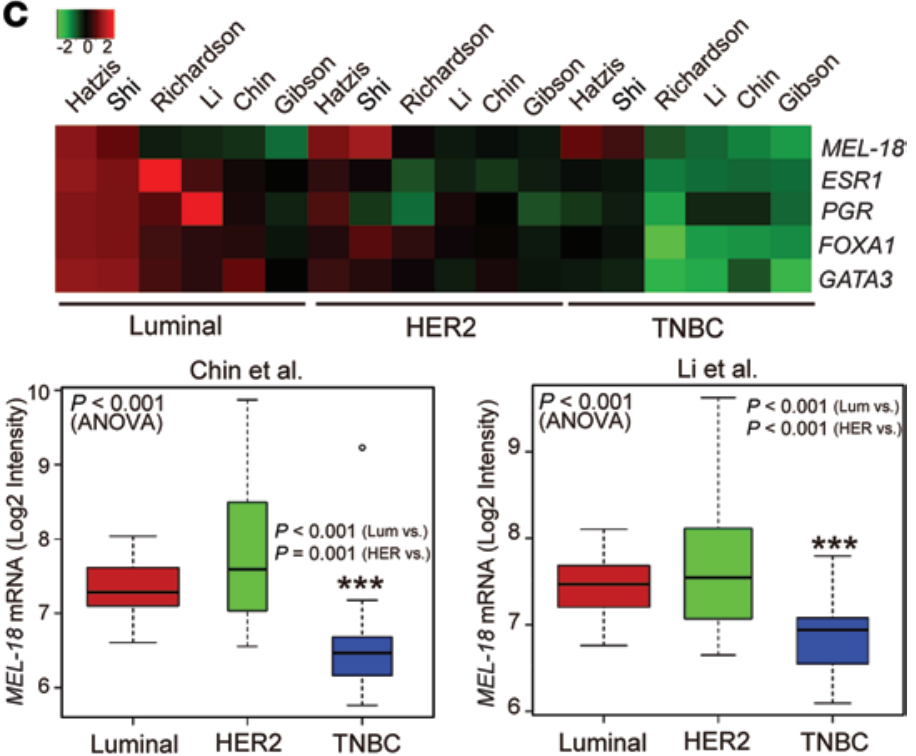

D
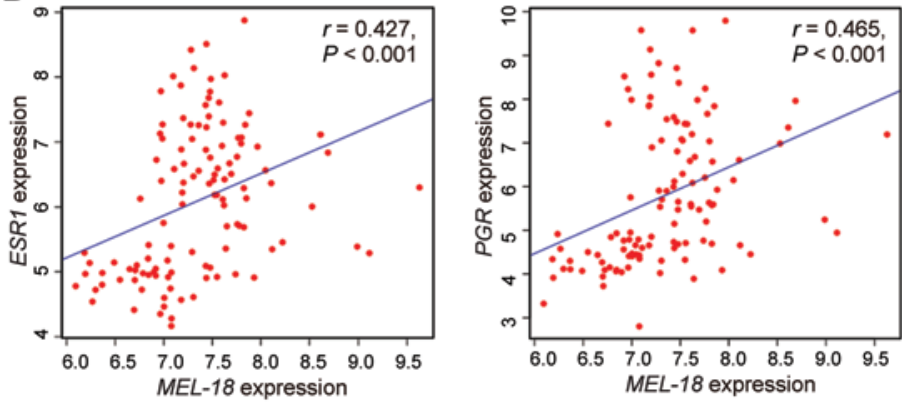

TNBC group $(n=53)$

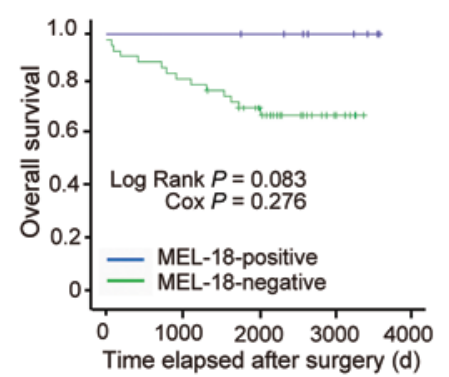

Figure 1. Loss of MEL-18 is associated with poor prognosis and TNBC. (A) The percentage of MEL-18 negativity and positivity in different breast cancer subtypes is shown as pie charts. ${ }^{*} P<0.01$ (Fisher's exact test). (B) Representative IHC images and bar graphs showing the correlation between MEL-18 expression and ER- $\alpha$ and PR expression in 223 breast tumor samples. Scale bars: $100 \mu \mathrm{m} .{ }^{*} P<0.05,{ }^{* *} P<0.01$ (Fisher's exact test). (C) Heatmap (top) and box plots (bottom) of MEL-18 mRNA levels in different breast cancer subtypes in the published microarray datasets from the indicated breast cancer cohorts (defined in Table 1). The bottom and top of the boxes correspond to the first and third quartiles; the bands inside the boxes represent the 50th percentile (median); the whiskers represent the lowest and highest values within 1.5-fold of the interquartile range (IQR) of the lower and upper quartiles; and the outliers are all values beyond the whiskers. $P$ values were calculated via ANOVA with pairwise comparisons. ${ }^{* *} P<0.001$ vs. luminal breast cancer (Lum). (D) Scatter plots showing the correlation of MEL-18 expression with ESR1 and PGR expression in a GEO dataset (CSE19615) (22). The $r$ value was calculated via Spearman's rank correlation coefficient analysis. (E) OS and DFS according to MEL-18 expression among 223 human breast cancer and 53 TNBC cases. The data were analyzed using the Kaplan-Meier method with the log-rank test and Cox regression model. ${ }^{*} P<0.05,{ }^{* *} P<0.01$.

of MCF-7 and T47D cells in which MEL-18 was knocked down compared with the xenografts of control cells, as measured by the tumor incidence and growth rates (Figure 3D, Table 3, Supplemental Figure 6 , and Supplemental Table 2). Moreover, IHC analysis demonstrated that the tumors formed in control mice in the absence of E2 treatment exhibited low expression of MEL-18, ER- $\alpha$, and PR, and that MEL-18 depletion further downregulated ER- $\alpha$ (Figure 3E).
Because ER- $\alpha$ loss is a major cause of antihormonal therapy resistance, we investigated the effect of MEL-18 on tamoxifen treatment in breast cancer cells. MEL-18 knockdown conferred tamoxifen resistance to luminal breast cancer cells, and notably, MEL-18 overexpression restored the sensitivity of TNBC cells to tamoxifen (Figure 4A and Supplemental Figure 7A). These effects were mediated by ER- $\alpha$ because the restoration of ER- $\alpha$ expression abolished 
Table 1. Correlation coefficients for the relationships between the mRNA expression of MEL-18 and that of 4 luminal markers (ESR1, PGR, FOXA1, and GATA3) in 7 independent microarray datasets

Dataset (accession no.)

Ref.

$n$

Richardson et al., 2006 (GSE7904)

Li et al., 2010 (GSE19615)

Shi et al., 2010 (GSE20194)

Hatzis et al., 2011 (GSE25066)

Chin et al., 2006 (E-TABM-158)

Cibson et al., 2005 (MDA-133)

Bos et al., 2009 (GSE12276)

$\begin{array}{ll}21 & 43 \\ 22 & 115 \\ 23 & 278 \\ 24 & 508 \\ 25 & 100 \\ 26 & 133 \\ 27 & 204\end{array}$

$\begin{array}{cc} & \text { ESR1 } \\ 43 & 0.285(0.025) \\ 15 & 0.427(<0.001) \\ 78 & 0.307(<0.001) \\ 08 & 0.386(<0.001) \\ 00 & 0.385(<0.001) \\ 133 & 0.291(<0.001) \\ 04 & 0.379(<0.001)\end{array}$

Comparison with MEL-18 expression $r$ value ( $P$ value)

$\begin{array}{ccc}\text { PCR } & \text { FOXA1 } & \text { CATA3 } \\ 0.400(0.001) & 0.448(<0.001) & 0.459(<0.001) \\ 0.465(<0.001) & 0.504(<0.001) & 0.505(<0.001) \\ 0.182(0.002) & 0.313(<0.001) & 0.239(<0.001) \\ 0.252(<0.001) & 0.492(<0.001) & 0.434(<0.001) \\ 0.255(0.005) & 0.447(<0.001) & 0.425(<0.001) \\ 0.155(0.074) & 0.399(<0.001) & 0.399(<0.001) \\ 0.363(<0.001) & 0.420(<0.001) & 0.446(<0.001)\end{array}$

The mean expression of the multiple probes for each gene on the Affymetrix GeneChips was used to determine the correlation coefficient. The $r$ value was calculated via Spearman's rank correlation coefficient analysis. Scale: +1 , perfect positive correlation; 0 , no correlation; and -1 , perfect inverse correlation. $r>0.3$ and $P<0.05$ indicated a significant positive relationship.

tamoxifen resistance in MEL-18-silenced MCF-7 cells (Supplemental Figure 7B). Moreover, the effect of transient ER- $\alpha$ knockdown by siRNA in control MCF-7 cells was similar to that of MEL-18 knockdown. Consistent with these in vitro results, tamoxifen treatment resulted in antiproliferative and antiapoptotic effects in mouse xenografts of MEL-18-silenced MCF-7 cells, as confirmed by growth curve analysis and both Ki-67 and TUNEL staining (Figure 4B and Supplemental Figure 8A). MEL-18 depletion also sustained reduced ER- $\alpha$ and PR expression, and increased AKT activity in these mice treated with E2 and/or tamoxifen (Figure 4C and Supplemental Figure 8A). Furthermore, mice bearing MEL-18-overexpressing MDAMB-468 TNBC cell tumors acquired sensitivity to tamoxifen treatment, although no difference in overall tumor growth was observed between the control and MEL-18-overexpressing cell xenografts (Figure 4D). In mice injected with MDA-MB-231 cells, which exhibit more rapid tumor growth than MDA-MB-468 cells in xenograft models, MEL-18 overexpression also slightly sensitized the tumors to tamoxifen and inhibited long-term tumor growth (Supplemental Figure 7C). Thus, these in vitro and in vivo data indicate that MEL18 affects the response of both luminal breast cancer and TNBC to tamoxifen. Several estrogen-independent growth factors and their downstream targets, such as those in the PI3K/AKT pathway, have been reported to contribute to antiestrogen resistance in breast cancer (30). Because we observed that AKT is consistently activated in MEL-18-silenced luminal breast cancer cells, regardless of treatment with E2 and/or tamoxifen (Supplemental Figure 5B and Supplemental Figure 8A), we further investigated whether $\mathrm{PI}$ KK/AKT signaling is involved in the MEL-18-mediated alteration of the tamoxifen response. In vivo treatment of xenograft tumors of MCF-7 cells with the PI3K inhibitor BKM120 revealed that the MEL-18-silenced MCF-7 cell-based tumors were more sensitive to BKM120 than to tamoxifen compared with control cell-based tumors (Supplemental Figure 8C). Moreover, combined treatment with BKM120 and tamoxifen resulted in a synergistic antitumor effect on these tumors both in vitro and in vivo (Supplemental Figure 8, B and C). Together, these results indicate that ER- $\alpha$ loss and AKT activation are required for MEL-18 loss-mediated tamoxifen resistance. We also confirmed that the subset of ER- $\alpha$-positive patients with negative MEL-18 expression who received tamoxifen $(n=103)$ exhibited significantly worse outcomes than those with positive MEL-18 expression, including poorer OS and DFS (OS, $P=0.034$ and 0.048 ; DFS, $P=0.033$ and 0.026 ; log-rank test and Cox regression, respectively) (Figure $4 \mathrm{E}$ ). Collectively, these findings imply that continuous ER- $\alpha$ downregulation and alternative growth signaling activation due to MEL-18 loss confers characteristics of hormone receptor-negative breast cancer by causing resistance to estrogen deprivation and antihormonal therapy.

MEL-18 inhibits the SUMOylation of $p 53$ and SP1 to induce ESR1 expression. We next investigated the molecular mechanism by which MEL-18 regulates ER- $\alpha$ transcription. The proximal promoter region of ESR1, which is regulated by MEL-18, contains binding sites for several epigenetic and transcription factors (31, 32). However, no significant difference in the expression of known epigenetic modifiers of the ESR1 gene and PcG protein - or the status of histone modifications, including H3K27me3 and DNA methylation - in the ESR1 promoter was observed between control and MEL-18-silenced cells (Supplemental Figure 9, A-C). The total expression of major ESR 1 transcription factors, including p53, SP1, and c-Jun, was also unchanged (Supplemental Figure 9D).

MEL-18 functions as an anti-SUMO E3 ligase by directly binding to both UBC9 and its substrate $(19,20)$, and the SUMOylation of transcription factors is often involved in transcriptional inhibition (33). Therefore, we hypothesized that MEL-18 may regulate ESR1 transcription via the inhibition of SUMOylation. We first determined whether $\mathrm{p} 53$ and SP1, which are reported to be direct targets of UBC9 $(34,35)$, are substrates of MEL-18. MEL-18 directly interacted with and inhibited the binding of SUMO-1 to the 553 protein but not the SP1 protein in vitro (Supplemental Figure 10, A-D), whereas the in vivo SUMOylation assay indicated that MEL-18 inhibits the conjugation of SUMO-1 to both p53 and SP1 (Supplemental Figure 10E). Moreover, endogenous SUMOylated p53 and SP1 proteins were inversely regulated by MEL-18 in both MCF-7 and MDA-MB-468 cells (Figure 5A), implying that MEL-18 may regulate SP1 SUMOylation via an indirect SUMOylation-regulating pathway. SUMOylated forms of CBP and c-Jun were not detected in these cells. These results imply that MEL-18 directly or indirectly inhibits p53 and SP1 SUMOylation. Because both p53 and SP1 are global transcriptional regulators, we determined the common tar- 
Table 2. Univariate and multivariate analyses of survival in breast cancer patients

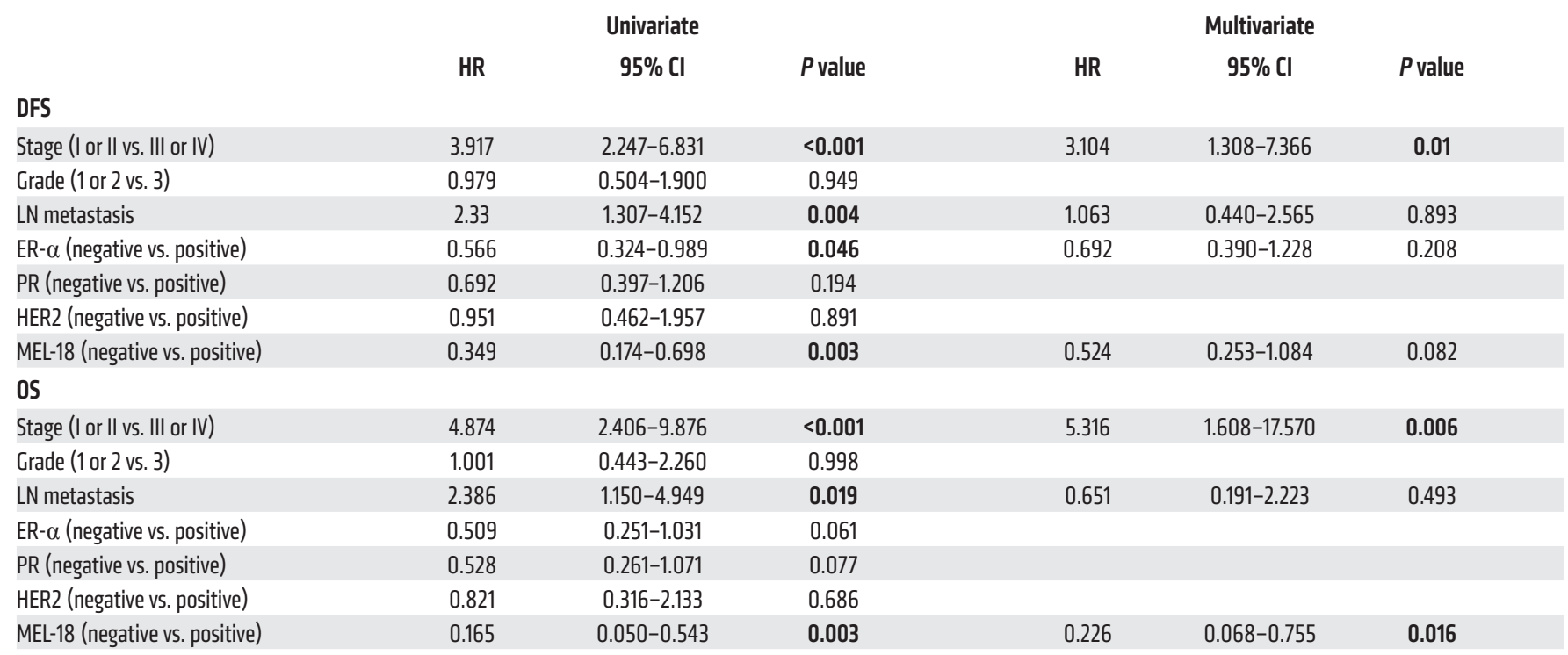

Cox regression hazard model. HR, hazard ratio. Boldface indicates significant $P$ values.

get genes of MEL-18 and p53/SP1 by comparing our microarray results for MEL-18-silenced MCF-7 cells with the gene expression profiles of a GEO dataset (GSE13291) (36) for MCF-7 cells treated with RITA, a small molecule that induces the cooperative activity of p53 and SP1 to transcriptionally regulate their target genes $(36,37)$. Several breast cancer subtype-specific markers and genes involved in transcriptional regulation and cell proliferation were common targets of MEL-18 and p53/SP1 (Figure 5B and Supplemental Figure 11A). Because ESR1 was a shared target gene in these datasets, we next determined whether p53 and SP1 SUMOylation influences ESR1 transcription. The activity of the proximal ESR1 promoter was augmented by the expression of SUMOylation-deficient mutant forms of p53 (K386R) or SP1 (E18A) compared with expression of their WT forms in MCF-7 cells (Supplemental Figure 11B). Furthermore, p53 K386R exhibited significant ESR1 promoter-binding ability compared with WT p53 (Supplemental Figure 11C). In TNBC cells carrying p53 point mutations (MDA-MB-468, R273H; MDA-MB-231, R280K), inhibition of SUMOylation via treatment with the SUMO inhibitor ginkgolic acid also enhanced ESR1 promoter activity (Supplemental Figure 11D). Similarly, the reduction in ESR1 promoter activity due to MEL-18 knockdown in MCF-7 cells was rescued by the expression of mutant forms of p53 or SP1 (Figure 5C). A synergistic effect was observed when the p53 and SP1 mutants were coexpressed. Consistent with this finding, in TNBC cells, silencing either p53 or SP1 using siRNA partially inhibited MEL-18-induced ESR1 promoter activity, and corepressing 553 and Sp1 completely abrogated this inhibition (Supplemental Figure 11E). Moreover, the recruitment of p53 and SP1 to the ESR1 promoter was inhibited by MEL-18 knockdown in luminal cells and was induced by MEL-18 overexpression in TNBC cells (Figure 5D). The binding of c-Jun and CBP, which were not detectably SUMOylated in these cells, to this promoter was unchanged by MEL-18 (Supplemental Figure 12A). In addition, the ability of p53 and SP1 to bind the promoter of CDKN1A, a major target gene of p53 that was not targeted by MEL-18 based on our microarray data, was reduced compared with ESR1 promoter binding in MCF-7 cells following MEL-18 knockdown (Supplemental Figure 12B), implying that ESR1 is a preferential target of the MEL-18mediated modulation of SUMOylation. We also confirmed that the reduction of p53 and SP1 SUMOylation induced by treatment with ginkgolic acid restored ER- $\alpha$ expression in MEL-18-silenced MCF-7 cells (Figure 5E). Taken together, these data imply that the SUMOylation of the transcription factors $\mathrm{p} 53$ and SP1 due to MEL18 loss impaired their recruitment to the ESR1 promoter, thereby reducing ER- $\alpha$ expression.

MEL-18 enhances deSUMOylation by inhibiting the ubiquitin-proteasome degradation of sentrin-specific protease 1. To further identify the mechanism by which MEL-18 regulates SUMOylation, the effect of MEL-18 on the expression of SUMO-related factors was examined. In MEL-18-silenced MCF-7 cells, the level of the 39-kDa SUMO-1conjugating form of the SUMO E2 enzyme UBC9 was enriched, whereas the level of the $18-\mathrm{kDa}$ free form of UBC9 was reduced (Supplemental Figure 13A). Conversely, MEL-18 overexpression increased the expression of the free form of UBC9 and SUMO-1 in TNBC cells. Notably, the expression and deSUMOylating enzyme activity of SUMO-1/sentrin-specific protease 1 (SENP1) were positively regulated by MEL-18 (Supplemental Figure 13, A and B). These data imply that MEL-18 inhibits SUMOylation by enhancing SENP1mediated deSUMOylation and by inhibiting UBC9-mediated SUMO-1 conjugation. We next examined the mechanism by which MEL-18 modulates SENP1 expression at the posttranscriptional level because the SENP1 mRNA level was not altered by MEL-18 (Figure 6A). We found that MEL-18 knockdown induced accelerated SENP1 protein degradation following treatment of MCF-7 cells with cycloheximide (CHX), a protein synthesis inhibitor (Figure 6B). Furthermore, treatment with the proteasome inhibitor MG132 restored 
A
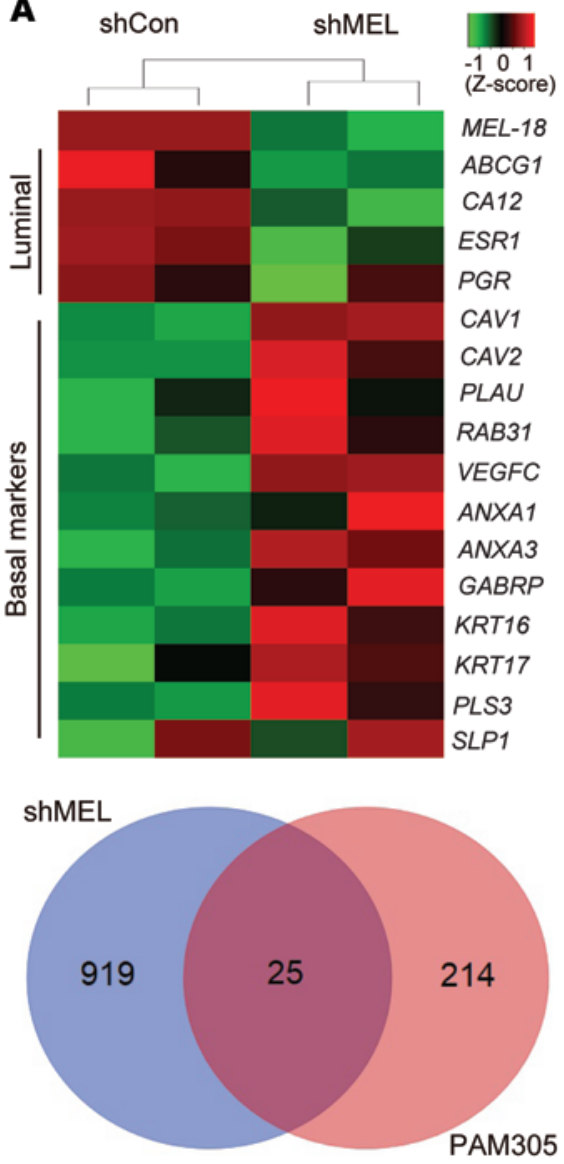

B

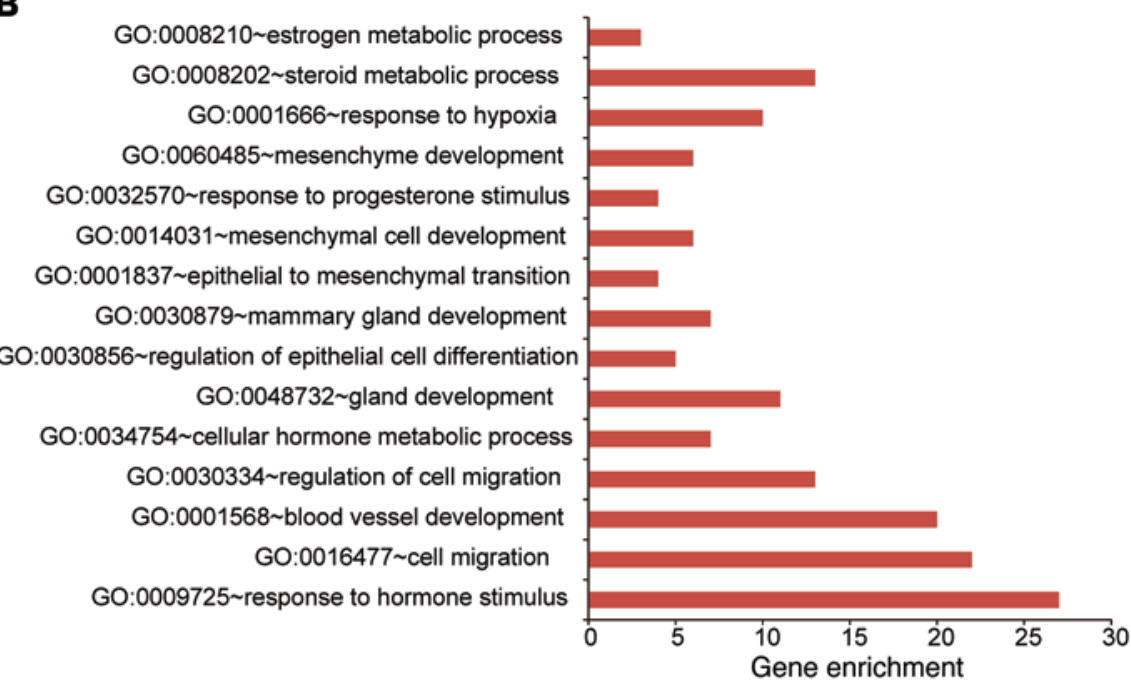

C

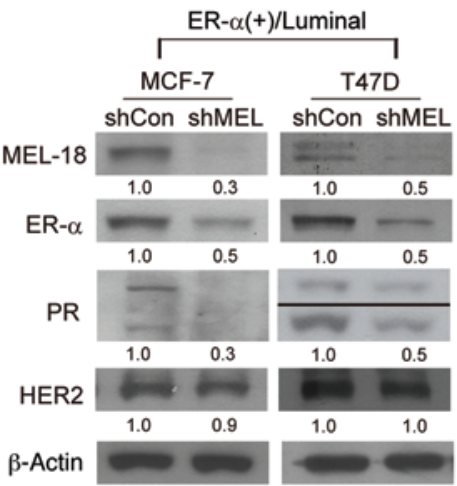

D

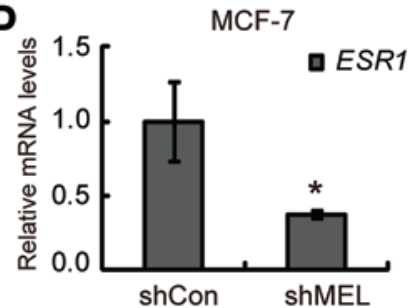

T47D

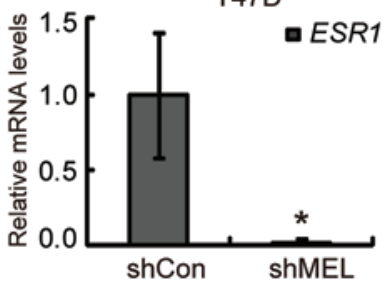

E

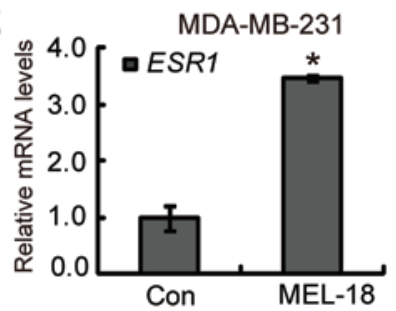

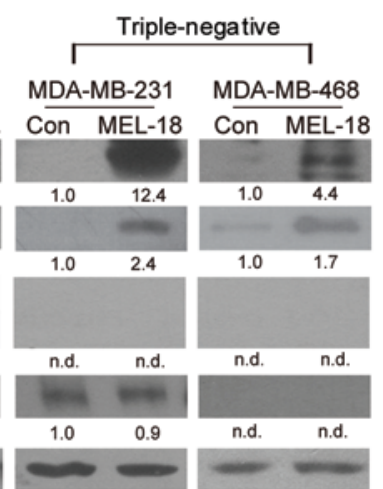

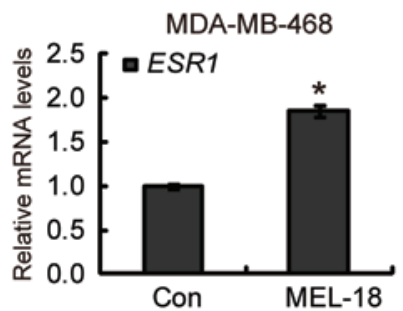

Figure 2. MEL-18 positively regulates ESR1 and PR expression. (A) Heatmap generated from the microarray analysis of MCF-7 cells expressing either control (shCon) or MEL-18 shRNA (shMEL) showing the differential expression of the luminal and basal markers between the two groups. The Venn diagram shows the number of common genes between the MEL-18 target genes and the PAM305 gene list. (B) The MEL-18 target genes obtained from the microarray analysis were categorized according to gene function via GO enrichment analysis. (C) The MEL-18-silenced (shMEL) or MEL-18-overexpressing (MEL-18) breast cancer cells and control cells (shCon and Con) were cultured in DMEM containing 10\% FBS for 48 hours, and the cell lysates were subjected to immunoblotting using the indicated antibodies. To detect ER- $\alpha$ protein expression in TNBC cells, more than $100 \mu \mathrm{g}$ of lysate was used for immunoblotting. The relative immunoblot band densities are indicated at the bottom of each blot. n.d., not detected. A black line within the blot indicates that the bands were spliced from the equal lane in the same gel because of the expression of isoforms of PR at different molecular weights (lower, PR-A, 81 kDa; upper, PR-B, $116 \mathrm{kDa}$ ). The data are representative of three independent experiments. ( $\mathbf{D}$ and $\mathbf{E}$ ) The mRNA levels of ER- $\alpha$ (ESR1) in the indicated stable cell lines were validated via qRT-PCR. The data represent the mean \pm SD of triplicate measurements. ${ }^{*} P<0.05$ vs. the controls (shCon or Con) based on 2-tailed Student's $t$ test.

SENP1 expression in these cells (Figure 6C), and MEL-18 blocked both exogenously and endogenously ubiquitinated SENP1 protein as measured by an in vivo ubiquitination assay (Figure 6, D and E). Thus, these results suggest that MEL-18 loss enhances the ubiquitinmediated proteasomal degradation of SENP1. To identify the molecular mechanism underlying SENP1 protein stabilization by MEL-18, we next investigated whether the BMI-1/RING1B ubiquitin ligase complex, which is negatively regulated by MEL-18 (18), targets the SENP1 protein. As shown in Figure 6F, the overexpression of a catalytically inactive mutant of RING1B (C51W/C54S), but not WT RING1B, restored the SENP1 protein level and consequently increased ER- $\alpha$ expression in MEL-18-silenced MCF-7 cells. Similar effects were observed when RING1B cofactor BMI-1 was silenced by siRNA in MCF-7 cells (Figure 6G), indicating that MEL-18 prevents the ubiquitin-mediated proteasomal degradation of SENP1 by inhibiting BMI-1/RING1B. 
A

MCF-7 (luminal)

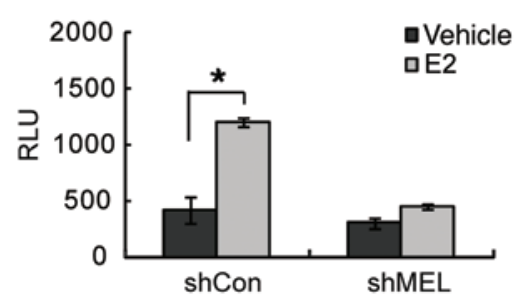

MDA-MB-468 (TNBC)

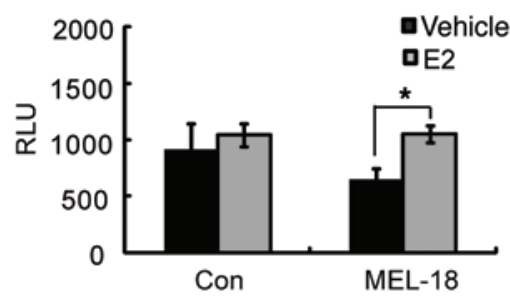

D

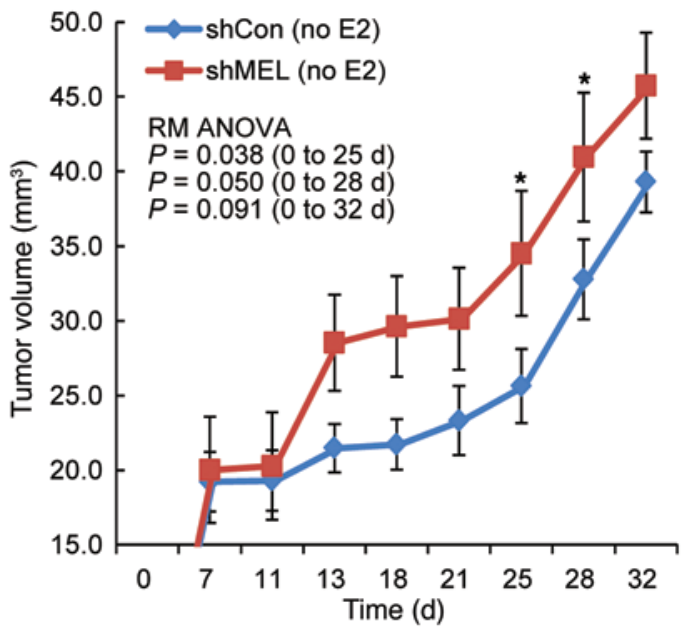

B
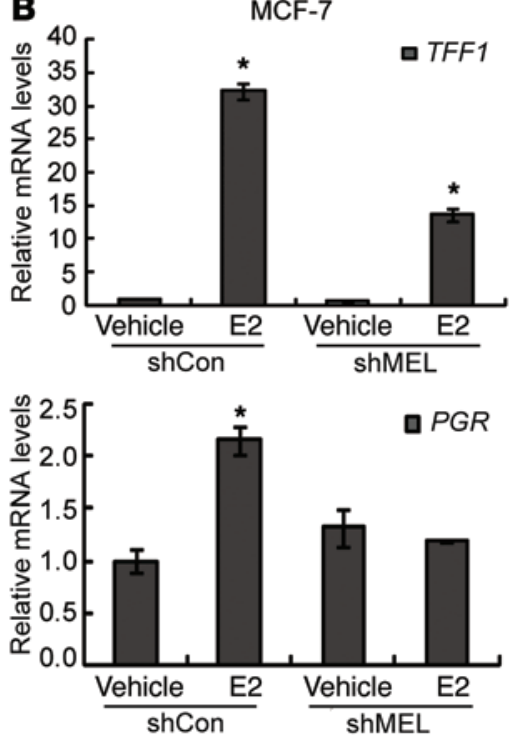

E
C
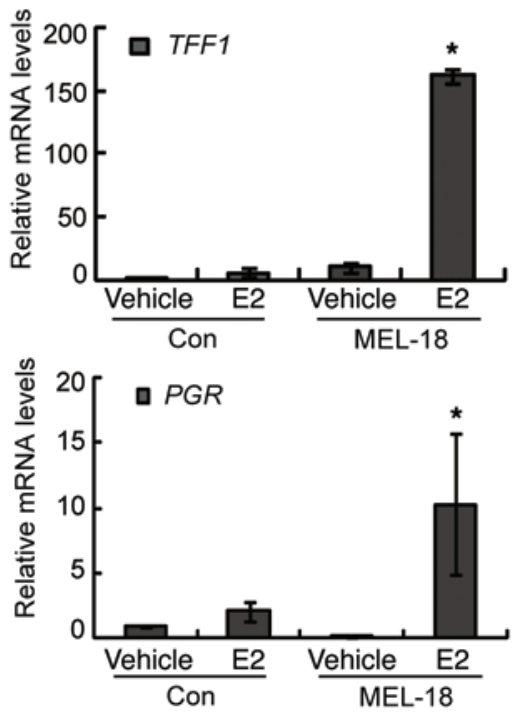

ER- $\alpha$
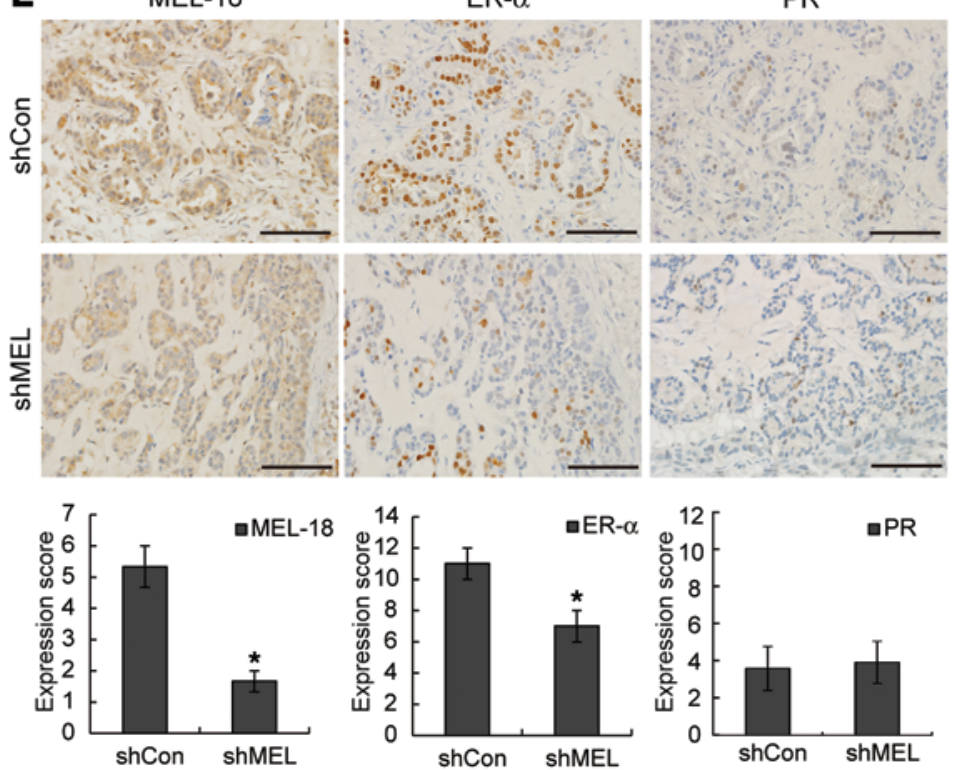

Figure 3. MEL-18 depletion abrogates ER- $\alpha$-dependent transcriptional activity and induces estrogen-independent tumor growth. (A-C) ERE luciferase assay (A) and qRT-PCR analysis of TFF1 (also known as pS2) and PR (PCR) expression levels (B and $\mathbf{C}$ ) in the control and MEL-18-silenced or MEL-18-overexpressing cell lines in the presence or absence of E2 (10 nM in MCF-7 cells or $20 \mathrm{nM}$ in MDA-MB-468 cells) for 24 hours. The error bars represent the mean \pm SD of triplicate experiments. ${ }^{*} P<0.05$ compared with the control (2-tailed Student's $t$ test). (D) The effect of MEL-18 knockdown on E2-independent breast tumor growth. Control or shMEL MCF-7 cells were transplanted into the mammary fat pads of NOD/SCID mice $(n=8)$ in the absence of E2 treatment. Tumor size was monitored to assess mouse xenograft tumor growth. ${ }^{*} P<0.05$ (group $\times$ days) based on RM ANOVA from day 0 to the indicated days. $P<0.001$ (days; RM ANOVA). (E) IHC for MEL-18, ER- $\alpha$, and PR in the indicated samples from three independent xenografted mice. Scale bars: $100 \mu \mathrm{m}$. The data in D and $\mathbf{E}$ are presented as the mean \pm SEM ( $n=8$ and $n=3$, respectively, independent experiments). ${ }^{*} P<0.05$ vs. shCon (2-tailed Student's $t$ test).

Because SENP1 overexpression reverses MEL-18 depletionmediated ER- $\alpha$ downregulation in MCF-7 cells (Figure 6H), we further verified the effects of SENP1 expression and its deSUMOylation activity on hormone receptor expression. SENP1 silencing by siRNA increased both p53 and SP1 SUMOylation, and subsequently downregulated ER- $\alpha$ and PR expression at the transcriptional level in WT MCF-7 cells (Supplemental Figure 14, A-C). Moreover, a catalytically inactive mutant of SENP1 (R630L/ K631M) impaired the deSUMOylation of p53 and SP1, and did not increase ESR1 or PGR transcription (Supplemental Figure $14, \mathrm{D}-\mathrm{F})$. These data demonstrate that MEL-18 enhances the
deSUMOylation of p53 and SP1 by inhibiting the ubiquitin-proteasomal degradation of SENP1 to regulate ER- $\alpha$ and PR expression.

We also confirmed the positive correlation of SENP1 protein expression with MEL-18 expression in 223 human breast tumors (Supplemental Figure 15A, $P=0.001$ ) and in a mouse xenograft model (Supplemental Figure 15, B and C). Furthermore, SENP1 negativity was associated with TNBC $(P=0.001)$, ER- $\alpha$ negativity $(P<0.001)$, higher histological grade $(P=0.010)$, and larger tumor size $(P=0.032)$ (Supplemental Table 3$)$. However, multivariate analysis indicated that SENP1 expression was not a strong independent prognostic factor for survival (Supplemental Table 4). 
Table 3. In vivo effect of MEL-18 knockdown on estrogen-independent MCF-7 tumor formation $(n=8)$

\begin{tabular}{|c|c|c|c|c|c|}
\hline \multirow[t]{2}{*}{ Group } & \multicolumn{5}{|c|}{ Tumor incidence ( $\geq 30 \mathrm{~mm}^{3}, n=8$ ) } \\
\hline & Day 7 & Day 13 & Day 21 & Day 28 & Day 32 \\
\hline shCon & $0 / 8(0 \%)$ & $0 / 8(0 \%)$ & $1 / 8(12.5 \%)$ & $6 / 8(75 \%)$ & $8 / 8(100 \%)$ \\
\hline ShMEL & $1 / 8(12.5 \%)$ & $4 / 8(50 \%)$ & $5 / 8(62.5 \%)$ & $7 / 8(87.5 \%)$ & $8 / 8(100 \%)$ \\
\hline
\end{tabular}

Tumor incidence rates in NOD/SCID mice injected with MCF-7 cells expressing either control (shCon) or MEL-18 shRNA (shMEL) in the absence of E2 injection. The significance of the difference in tumor incidence was determined via Poisson distribution analysis $(P<0.001)$.

Therefore, these data indicate the importance of MEL-18-mediated SENP1 regulation in breast cancer progression and suggest SENP1 and MEL-18 as markers of ER- $\alpha$ negativity.

\section{Discussion}

In this study, we demonstrated that MEL-18 serves as a predictor of triple negativity and antihormonal therapy resistance in human breast cancer, and modulates hormone receptor expression. Furthermore, we identified a mechanism by which MEL-18 regulates the ESR1 gene. MEL-18 inhibits the SUMOylation of the ESR1 transcription factors $\mathrm{p} 53$ and SP1 by repressing SUMO-1 conjugation or by activating deSUMOylation via the ubiquitin-proteasomal degradation of SENP1, enhancing the transactivation of ESR1 and consequently upregulating the PR (Figure 7A). Together, these results indicate that SUMO-mediated hormone receptor regulation by MEL-18 is crucial for breast cancer progression.

Our data suggest that the MEL-18 loss-induced lack of hormone receptor dependence is an important event in the progression of breast cancer (Figure 7B). MEL-18 loss may activate alternative estrogen-independent signaling pathways to gain hormone independence. The overexpression of receptor tyrosine kinases (RTKs) or the activation of signaling pathways downstream of RTKs, such as the PI3K/AKT pathways, is closely linked to estrogen independence and endocrine therapy resistance in breast cancer $(30,38)$. Indeed, our data demonstrate that MEL-18 depletion sustains increased AKT phosphorylation levels regardless of hormone or antihormone treatment, and that the inhibition of the PI3K/ AKT pathway abolishes MEL-18 loss-mediated tamoxifen resistance. Thus, we suggest that persistent ER- $\alpha$ downregulation and RTK-related signaling activation due to MEL-18 loss may confer estrogen independence and antiestrogen therapy resistance. Our recent studies have suggested that MEL-18 loss induces the expansion of the $\mathrm{CD} 44^{+} \mathrm{CD} 24^{-}$stem-like cell population and EMT, which are characteristics of hormone-independent basal-like breast cancer $(12,15)$. Furthermore, studies have demonstrated that MEL-18 is strongly involved in the regulation of several molecules that are associated with basal-like cancer or TNBC, including p53, PTEN, PI3K, Wnt, Notch, and miR-205 $(12,13,15)$. For example, a high percentage of TNBC cases lack PTEN expression $(39,40)$, and we have demonstrated that MEL-18 loss induces PTEN downregulation and PI3K/AKT activation (13). The loss of p53 function is characteristic of TNBC (41). In this study, MEL-18 was associated with p53 function by activating p53 transcription, even when p53 was expressed in mutant form in TNBC cells. These pieces of evidence also suggest that MEL-18 may play a critical role in the regulation of luminal and basal-like cell fates in mammary glands; this possi- bility should be further examined using a genetically engineered mouse mammary tumor virus (MMTV) model. Together, these findings suggest that the loss of MEL-18 function in hormonedependent breast cancer exacerbates the aggressiveness of breast cancer by altering the expression or activity of hormone receptors.

We here provide the first evidence to our knowledge for the SUMOylation-related transcriptional regulation of ESR1 by MEL-18. Although the effects of SUMOylation on p53 transcriptional activity are controversial, our findings and those of other recent studies indicate that p53 SUMOylation is associated with a loss of its transcriptional activity $(42,43)$. p53 forms a transcriptionally active complex with other transcription factors, including SP1, c-Jun, and CBP, to regulate ESR1 transcription (32). Our data indicate that p53 and SP1 are independent transcriptional activators of ESR1 and that the modulation of the SUMOylation of both p53 and SP1 by MEL-18 exerts a synergistic effect on ESR1 promoter regulation, even though this modification of SUMOylation does not affect the binding of c-Jun or CBP to the ESR1 promoter. Thus, these findings indicate the importance of the MEL-18-mediated modification of the p53 and SP1 proteins in the regulation of ESR1 transcription. Because mutant p53 (R273H and R280K) retains its function, targeting the SUMOylation pathway to modulate ESR1 transcription may be effective in both WT and mutant p53-expressing cells. Furthermore, we elucidated the mechanism by which MEL-18 regulates the SUMOylation process. In addition to previous data indicating that MEL-18 directly inhibits SUMOylation via the binding partner $\operatorname{UBC} 9(19,20)$, our findings reveal that MEL-18 is also involved in the indirect regulation of SUMOylation via the modulation of SENP1 protein expression. Furthermore, we observed that SENP1 is a ubiquitination target of the MEL-18-containing PRC-1 complex and is also crucial for ESR1 regulation by enhancing the deSUMOylation of the transcription factors of ESR1. However, SENP1 is less clinically relevant than MEL-18 based on survival analysis, possibly because SENP1 is a downstream target of MEL-18 in the regulation of ER- $\alpha$. We further expect that inhibiting SUMOylation via MEL-18 and SENP1 overexpression in combination with epigenetic agents may enhance ESR1 re-expression in hormone receptor-negative breast cancer therapy.

Our data support the clinical significance of MEL-18 in human breast cancer. Previous studies, including our own, have demonstrated that MEL-18 expression is lower in human breast cancer tissues than in normal breast tissues $(9,10,14)$, but the clinical importance of MEL-18 in different breast cancer subtypes has not been evaluated. In this study, based on IHC analysis of breast cancer specimens and the gene expression profiles of multiple independent microarray datasets from various breast cancer cohorts, we demonstrated a negative correlation of MEL-18 
A

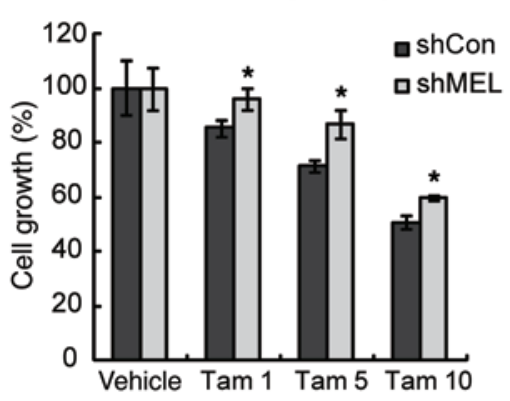

MDA-MB-468 (TNBC)

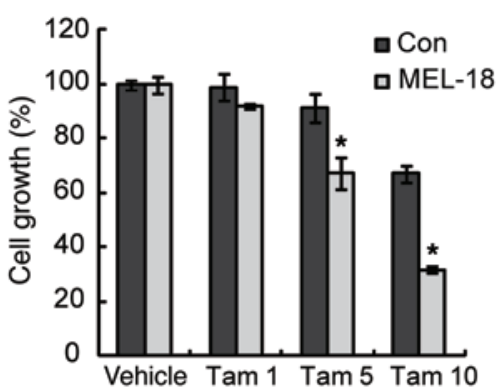

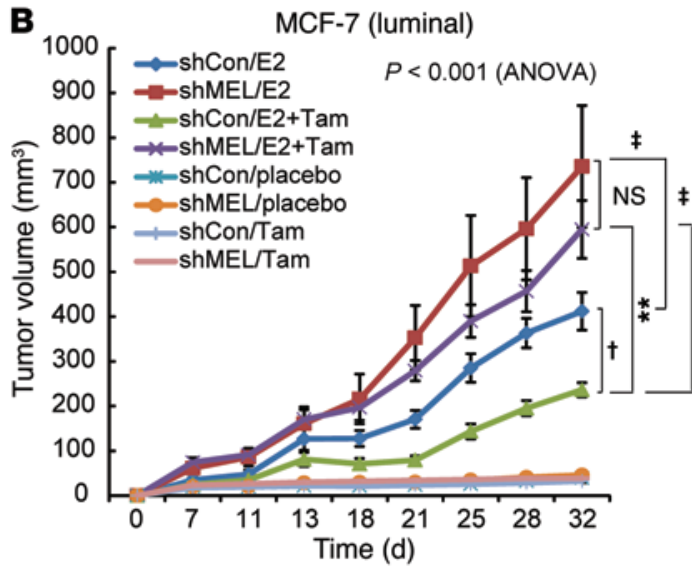

C

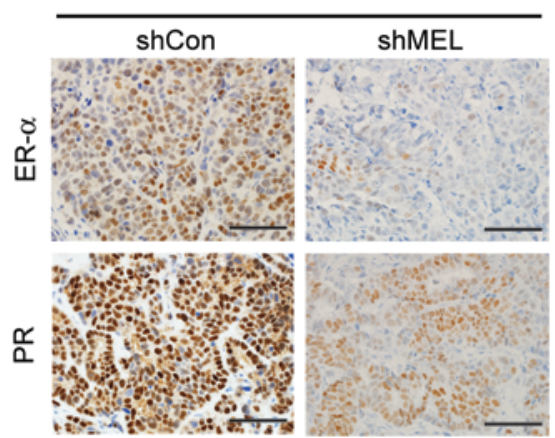

D

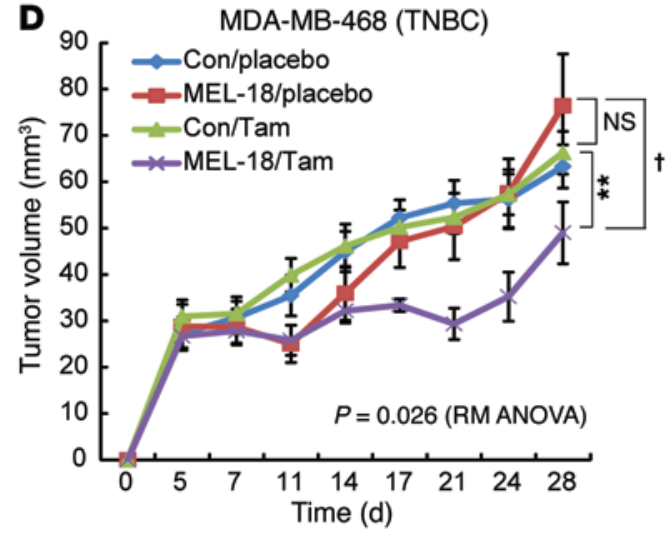

E2+Tam

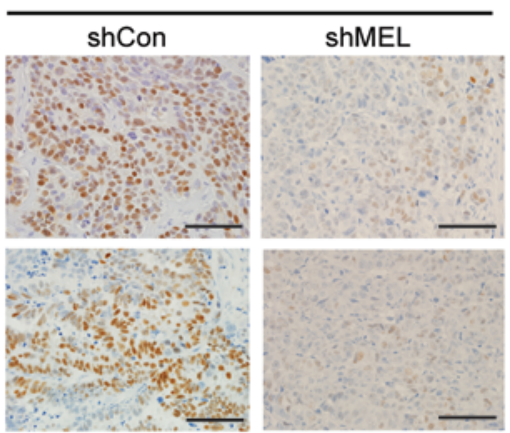

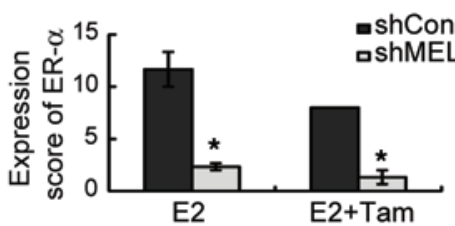

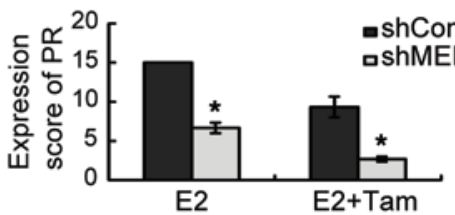

E

ER- $\alpha$-positive \& Tamoxifen Tx. group $(n=103)$
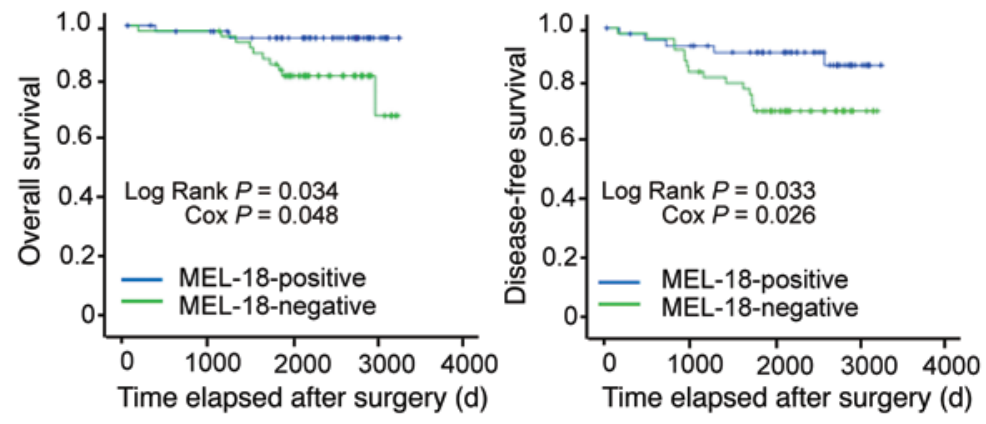

Figure 4. The loss of MEL-18 induces resistance to antiestrogen therapy. (A) Cell viabilities following treatment with the indicated doses ( $\mu \mathrm{M}$ ) of tamoxifen (Tam) or ethanol (vehicle) for 5 days were analyzed via the MTT assay. The data are presented as mean \pm SD $(n=3)$. (B) NOD/SCID mice injected with control or MEL-18-silenced cells following implantation with or without E2 pellets were administered Tam for 4 weeks ( $n=8$ per group; mean \pm SEM). $P$ values for multiple comparisons (4 groups: shCon/E2, shMEL/E2, shCon/E2+Tam, and shMEL/E2+Tam) were calculated via Welch ANOVA followed by Dunnett's T3 test. ${ }^{* *} P=0.004$ vs. shCon/E2+Tam; ${ }^{\dagger} P=0.019$ vs. shCon/E2; $P=$ NS vs. shMEL/E2; ${ }^{\ddagger} P<0.001$ (shCon/E2+Tam vs. shMEL/E2+Tam) and $P=0.043$ (shCon/E2 vs. shMEL/E2) based on RM ANOVA. (C) IHC for ER- $\alpha$ and PR in the xenografted tumors from the indicated groups of mice (mean \pm SEM of 3 mice). ${ }^{*} P<0.05$ vs. shCon (2-tailed Student's $t$ test). Scale bars: $100 \mu \mathrm{m}$. (D) Tumor growth curves for NOD/SCID mice implanted with control or MEL-18-overexpressing MDA-MB-468 cells treated with Tam $(5 \mathrm{mg} /$ pellet) or placebo $(n=8$ per group; mean $\pm \mathrm{SEM})$. $P<0.001$ (days), $P=0.026$ (group $\times$ days) based on RM ANOVA. ${ }^{* *} P=0.006$ vs. Con $/$ Tam; ${ }^{\dagger} P=0.026$ vs. MEL-18/placebo (post hoc LSD test). (E) Analysis of OS and DFS according to MEL-18 expression in 103 Tam-treated ER- $\alpha$-positive human breast tumors using the Kaplan-Meier method.

protein and mRNA levels with the TNBC subtype. Furthermore, although the correlation between the expression of MEL-18 and hormone receptors remains controversial, as Guo et al. failed to detect any association between MEL-18 and ER- $\alpha$ expression in their breast cancer cohort (10), our data and these microarray datasets reveal a significant correlation of MEL-18 expression with the expression of ER- $\alpha$ and other luminal markers in human breast cancer. Our clinical data also demonstrated that MEL-18 is a more valuable prognostic marker of luminal breast cancer than of TNBC. The lack of a statistically significant difference in survival between the low and high MEL-18 expression groups of patients with TNBC or basal breast cancer subtypes may be due to the exclusive downregulation of MEL-18 and either the lack of its downstream target ER- $\alpha$ in the TNBC group or the molecular heterogeneity and aggressive characteristics of TNBC. Notably, breast cancer patients displaying MEL-18 loss were less likely to 
A

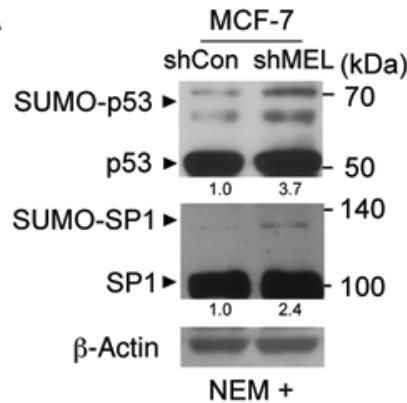

C

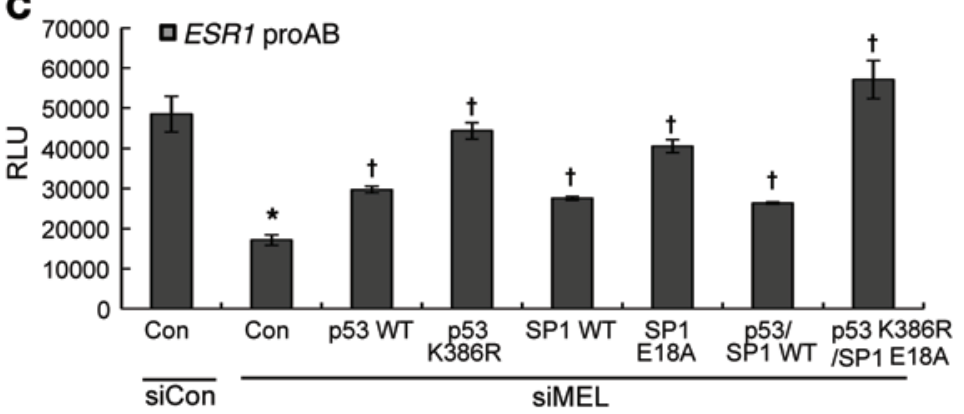

D
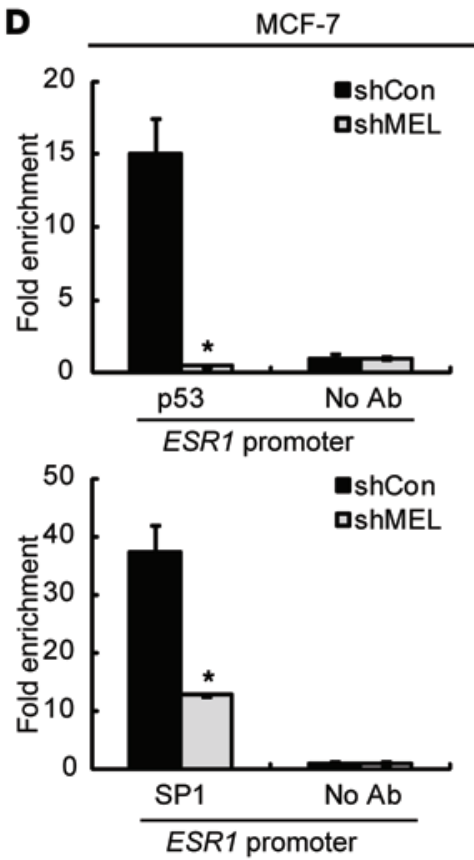

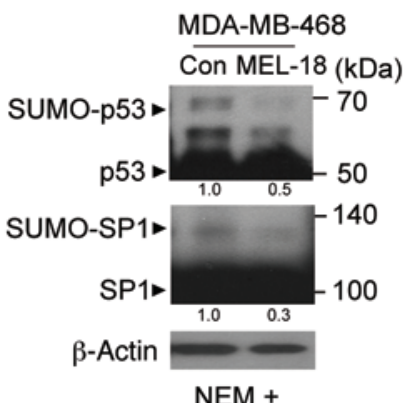

$\mathrm{NEM}+$
B

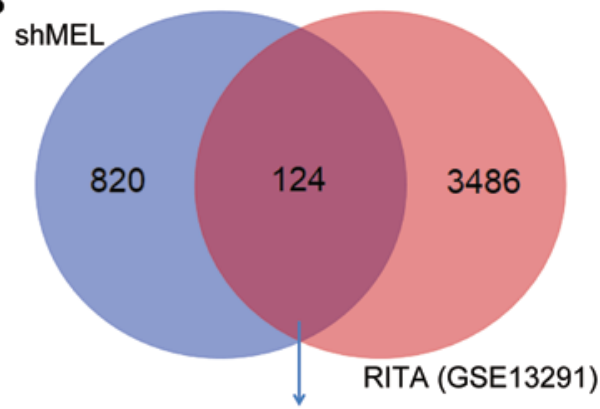

\begin{tabular}{|l|l|}
\hline Luminal & Basal \\
\hline CA12 & CAV1 \\
ESR1 & CD44 \\
& FXYD5 \\
& IGFBP6 \\
& MGP \\
\hline
\end{tabular}

E
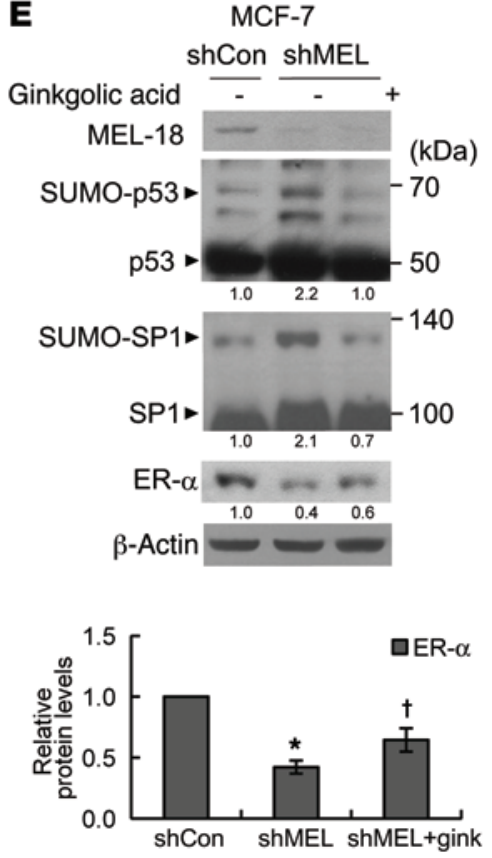

Figure 5. MEL-18 regulates ESR1 transcription by inhibiting the SUMOylation of the ESR1 transcription factors p53 and SP1. (A) Cell lysates treated with $20 \mathrm{mM} \mathrm{N}$-ethylmaleimide (NEM) were subjected to immunoblotting. The amount of SUMOylated protein was quantified by measuring the ratio of SUMOylated protein/total protein. (B) Venn diagram showing the relationship between the microarray results for MCF-7 cells expressing MEL-18 shRNA (shMEL) and those for MCF-7 cells treated with RITA (GSE13291) (36). (C) MCF-7 cells expressing MEL-18 siRNA (siMEL) were cotransfected with WT or SUMOylation-deficient mutant constructs of $\mathrm{p} 53$ or SP1 and with ESR1 pro-Luciferase and were subjected to a luciferase reporter assay. The data are presented as the mean $\pm \mathrm{SD}(n=3)$. ${ }^{*} P<0.05$ vs. siCon/Con; ${ }^{\dagger} P<0.05$ siMEL/Con (2-tailed Student's $t$ test). (D) ChIP-qPCR analysis showing the amount of ESR1 transcription factor that was recruited to the ESR1 promoter in the indicated cells. The data are presented as the mean $\pm S D(n=3)$. ${ }^{*} P<0.05$ vs. shCon (2-tailed Student's $t$ test). (E) The effect of ginkgolic acid on the expression of ER- $\alpha$ in the MEL-18-silenced cells. Cells were treated with 100 mM ginkgolic acid for 24 hours and subjected to immunoblotting. Parallel samples examined on separate gels are shown. The data were quantified by measuring the immunoblot band densities from three independent experiments (mean $\pm \mathrm{SD}$ ). ${ }^{*} P<0.05$ vs. shCon; ${ }^{\dagger} P<0.05$ vs. shMEL (2-tailed Student's $t$ test). All data shown are representative of three independent experiments.

benefit from tamoxifen therapy. Resistance to endocrine therapy is a major complication of breast cancer treatment, and ER- $\alpha$ is currently the most important clinical biomarker for predicting the outcome of endocrine therapy (30). Several studies have demon- strated that ER- $\alpha$ downregulation leads to tamoxifen resistance in ER- $\alpha$-positive breast cancer $(44,45)$. A clinical trial aiming to reactivate ER- $\alpha$ and restore the benefits of tamoxifen to patients with TNBC (ClinicalTrials.gov, NCT01194908) is ongoing. Con- 
A

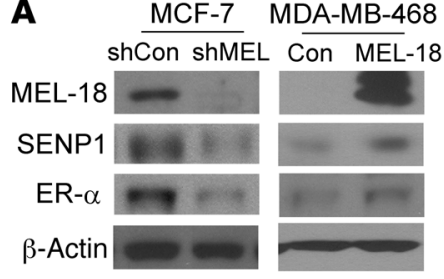

MCF-7
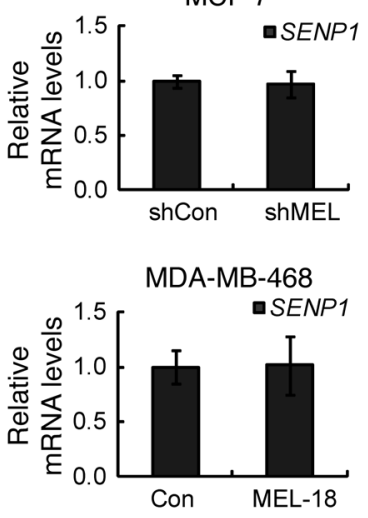

$\mathbf{E}$

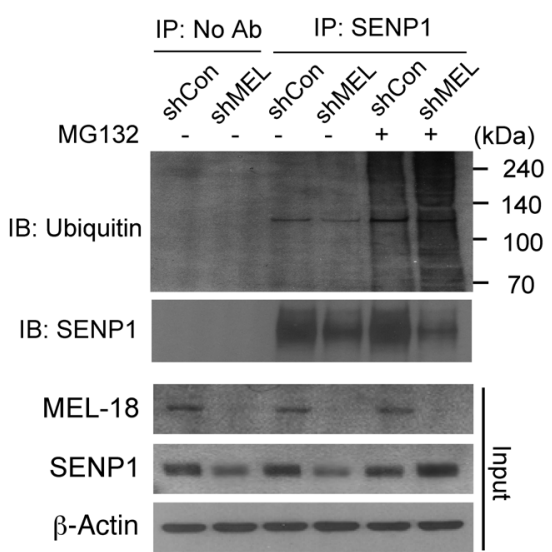

B
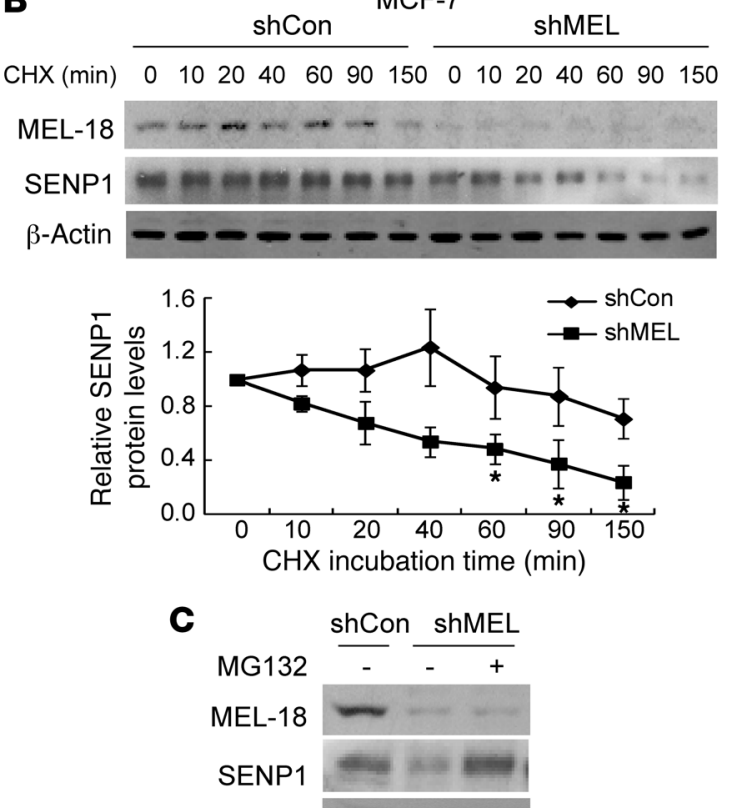

$\rightarrow$ shCon

$\rightarrow-$ shMEL

$\beta$-Actin

$\mathbf{F}$

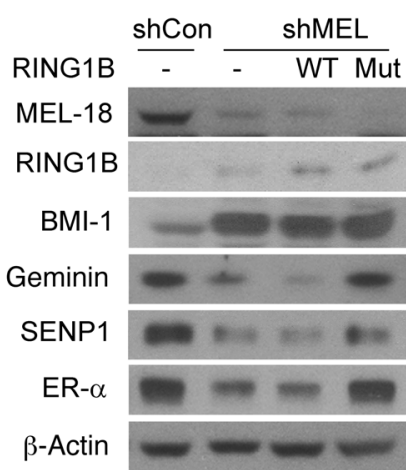

D

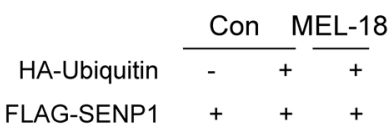

FLAG-SENP1 $+++(\mathrm{kDa})$

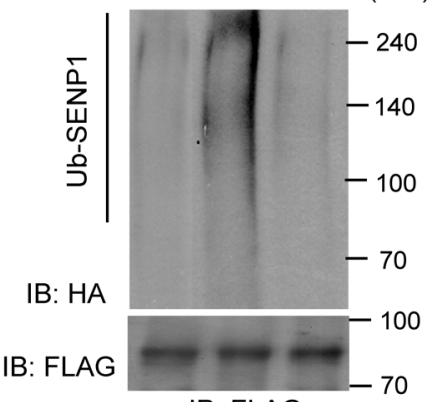

IP: FLAG

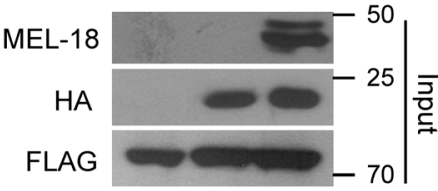

Figure 6. MEL-18 enhances the deSUMOylation of ESR1 transcription factors by inhibiting the ubiquitin-proteasomal degradation of SENP1. (A) Analysis of SENP1 expression via immunoblotting and qRT-PCR. (B and C) Immunoblotting of the cell lysates from the control and MEL-18-silenced MCF-7 cells treated with $100 \mu \mathrm{g} / \mathrm{ml}$ CHX for the indicated periods (B) or with DMSO or $10 \mu \mathrm{M}$ MG132 for 2 hours (C). The quantification of SENP1 protein stability is shown as a graph. The data in $\mathbf{A}$ and $\mathbf{B}$ are presented as the mean \pm SD of triplicate measurements. ${ }^{*} P<0.05$ vs. shCon ( 2 -tailed Student's $t$ test). (D) In vivo SENP1 ubiquitination assay in 293T cells. (E) Endogenous SENP1 protein ubiquitination levels in the control and MEL-18-silenced MCF-7 cells treated with or without $40 \mu \mathrm{M}$ MG132 for 6 hours. (F-H) Immunoblotting of the indicated cell lines. Cells stably expressing WT RING1B or a catalytically inactive RING1B mutant (Mut) (F) or SENP1 (H) were generated from MEL-18-silenced MCF-7 cells. For BMI-1 knockdown, nontargeted or BMI-1 siRNA was transfected into MEL-18-silenced MCF-7 cells for 48 hours (G). Ceminin protein, a known RING1B E3 ligase substrate, was used as a positive control for the measurement of RINC1B activity. All data are representative of three independent experiments.

sistent with this finding, our data demonstrated that MEL-18 overexpression confers tamoxifen sensitivity to TNBC cells, supporting the potential of MEL-18 as a therapeutic target to restore the sensitivity of TNBC to antihormonal therapy. Collectively, our clinical data strongly suggest that MEL-18 acts as a critical tumor suppressor and a regulator of ER- $\alpha$, and serves as a new prognostic marker of the outcome of tamoxifen treatment.

In summary, we demonstrated that MEL-18 functions as a regulator of SUMO-dependent hormone receptor transcription, and is a novel prognostic marker and predictor of the response to antihormonal therapy and of TNBC. Thus, MEL-18 may represent a promising molecular target to alter the resistance of breast cancer to antihormonal therapy.

\section{Methods}

Cell culture and drug treatment. All cell lines were purchased from ATCC. Human breast cancer cell lines were cultured in DMEM lacking phenol red (Welgen Inc.) containing 10\% FBS. The 293T cell lines were cultured as previously described (13). CHX and MG132 were obtained from Calbiochem, and ginkgolic acid, E2, 4-hydroxytamoxifen, R5020, and LY294002 were obtained from Sigma-Aldrich. For E2 and 4-hydroxytamoxifen treatment, cells were cultured in phenol red-free DMEM sup- 
A

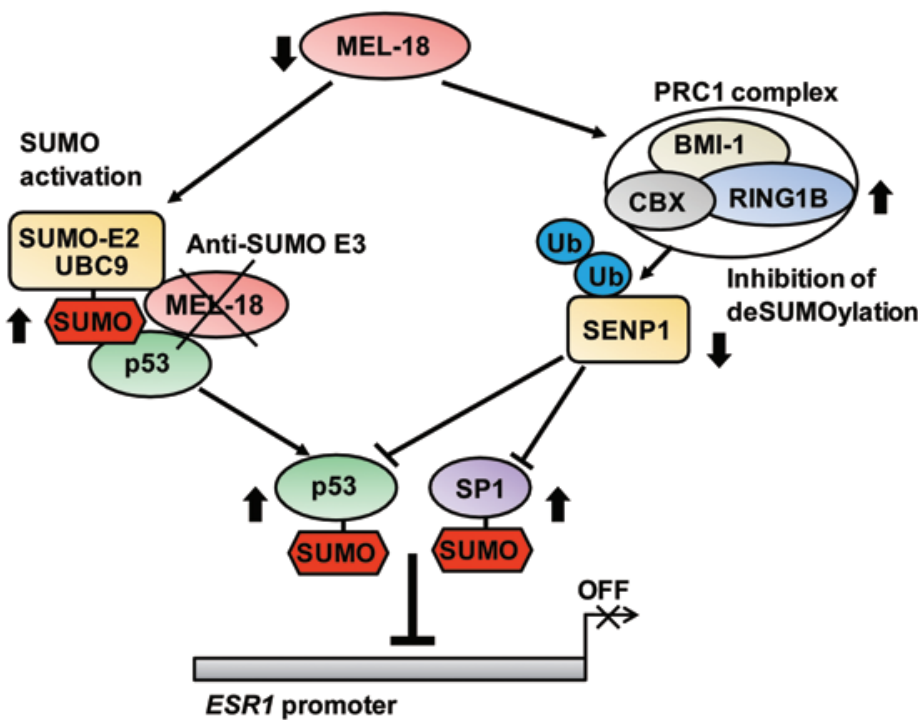

B

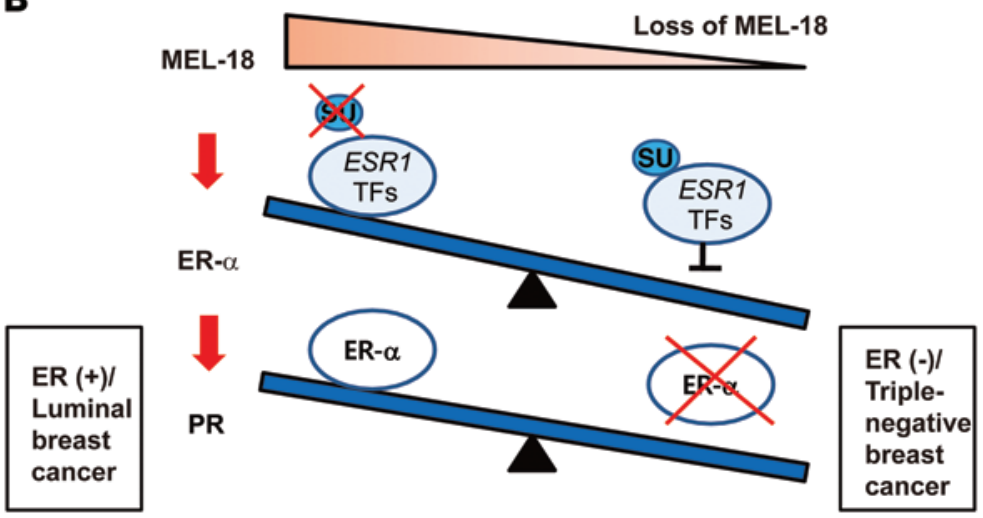

Figure 7. Proposed models for the regulation of hormone-dependent breast cancer by MEL-18. (A) Schematic model of the regulation of SUMO-dependent ER- $\alpha$ transcription by MEL-18. The loss of MEL-18 enhances SUMO activation via direct binding between the SUMO E2 enzyme UBC 9 and its substrate. Moreover, MEL-18 depletion inhibits the deSUMOylation activity of SENP1 by enhancing the BMI-1/RING1B E3 ubiquitin ligase complex-mediated ubiquitin-proteasomal degradation of SENP1. Via these two pathways, MEL-18 inhibits the SUMOylation of $\mathrm{p} 53$; alternatively, MEL-18 modulates SP1 SUMOylation via the SENP1-mediated deSUMOylation pathway. Increasing p53 and SP1 SUMOylation via MEL-18 silencing inhibits their recruitment to the ER- $\alpha$ promoter and downregulates ER- $\alpha$ expression. (B) Proposed model for the regulation of the balance between hormone dependence and independence by the polycomb protein MEL-18 in human breast cancer. In luminal breast cancer, MEL-18 contributes to the maintenance of the expression of the hormone receptors ER- $\alpha$ and PR but not HER2 by inhibiting the SUMOylation of ER- $\alpha$ transcription factors and by enhancing ER- $\alpha$-dependent transcriptional activity, respectively. However, when MEL-18 expression is lost during breast cancer progression, the tumor develops hormone independence and resistance to antihormonal therapy, phenotypes of hormone receptornegative breast cancers, including TNBC, which is characterized by the loss of ER- $\alpha$ and PR expression and the lack of HER2 amplification. Therefore, MEL-18 acts as a modulator of hormone receptor expression and a critical determinant of hormone dependence and independence in human breast cancer. SU, SUMOylation; TFs, transcription factors.

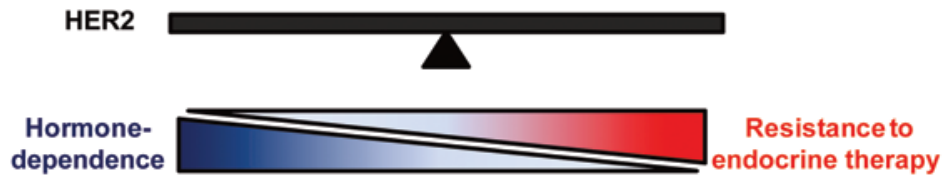

plemented with $10 \%$ charcoal/dextran-treated FBS. BKM120 (A-1108), a pan-PI3K inhibitor, was purchased from Active Biochemicals.

Gene expression microarray analysis. Total RNA was isolated from MCF-7 cells expressing control or MEL-18 shRNA to generate cDNA and multiple copies of biotinylated cRNA using the Illumina TotalPrep RNA amplification kit (Ambion). After purification, cRNA was hybridized to a HumanHT-12 v4 Expression BeadChip (Illumina) at $58^{\circ} \mathrm{C}$ for 16 hours via a multiple-step procedure according to the manufacturer's instructions. The chips were then washed, dried, and scanned on the Bead Array Reader, and the raw data were extracted using GenomeStudio version 2011.1 software (Illumina). Present/absent calling relied on a classification method based on detection $P$ values as calculated by GenomeStudio (Illumina). Genes with detection $P$ values $<0.05$ were classified as present, and all other genes were classified as absent. The selected gene signal values were logarithmically transformed and normalized using the quantile method. All genes displaying absolute fold changes of at least 1.5 between the compared groups were considered differentially expressed. These data were deposited in the Gene Expression Omnibus (GEO) with the accession number GSE64716. Further analysis was performed using the Database for Annotation, Visualization, and Integrated Discovery (DAVID; http://david.abcc.ncifcrf.gov/) to functionally categorize the genes involved in different biological processes. The PAM305 gene list for the classification of breast cancer cell lines into luminal and basal subtypes (46), as well as the gene expression microarray profiles of MCF-7 cells treated with the p53 reactivator RITA (GSE13297) from GEO (36), were used for comparison with the MEL-18 target genes identified based on our microarray data.

Data collection and analysis of the microarray datasets from the breast cancer patient cohorts. Seven publicly available datasets of human breast cancer gene expression microarrays were downloaded from the GEO database (http://www.ncbi.nlm.nih.gov/geo: GSE7904, ref. 21; GSE19615, ref. 22; GSE20194, ref. 23; GSE25066, ref. 24; GSE12276, ref. 27), ArrayExpress (http://www.ebi.ac.uk/arrayexpress: E-TABM-158, ref. 25), and MD Anderson (http://bioinformatics.mdanderson.org/pubdata.html: MDA133, ref. 26) and re-analyzed. The expression profiles of MEL-18, ESR1, PGR, FOXA1, and GATA3 were extracted from the independent datasets, and the relationship between the expression of MEL18 and each of these genes was determined by calculating the correlation 
coefficient ( $r$ value). The subtype classification of breast cancer based on ER, PR, and HER2 status (luminal, ER ${ }^{+}$HER2; HER2, ER ${ }^{+} \mathrm{HER}^{+}$or ER-HER2 $^{+}$; and TNBC, ER-PR-HER2-) was further performed for 6 of the 7 datasets for which IHC results were available for all 3 markers to compare the MEL-18 expression patterns in different breast cancer subtypes. For metaanalysis of the relationship between $M E L-18$ mRNA expression and the OS and DFS of breast cancer patients, Kaplan-Meier Plotter (http://kmplot.com/analysis) was used as described previously (28).

ChIP assay. ChIP assays were performed according to the manufacturer's instructions provided in the ChIP assay kit (Upstate Biotechnology). The binding of transcription factors to target promoters was detected using primers specific to the proximal promoter of ESR1 (5'-CGCTCCAAATCGAGTTGTGCCT-3' and 5'-CCGGGCCTCCAACTTTAAGTACTGG-3') and the CDKN1A (p21) promoter (5'-GCTGTGGCTCTGATTGGCTTT-3' and 5'-ACAGGCAGCCCAAGGACAAA- $3^{\prime}$ ). The primers targeting the epigenetic modification region of the ESR1 promoter were described previously (31). The enrichment of the ChIP signal was validated via quantitative real-time PCR (qRT-PCR) (signal/input ratio).

SUMOylation/deSUMOylation assay. In vitro SUMOylation was assessed according to the manufacturer's instructions for the SUMOlink SUMO-1 Kit (40120, Active Motif). Recombinant GST-MEL-18 protein was obtained from Novus Biologicals. Recombinant p53 and SP1 proteins (PR-733) were obtained from Active Motif and Jena Biosciences, respectively. For the in vivo SUMOylation assay, 293T cells were transfected for 36 hours. The cell extracts were immunoprecipitated using an anti-SUMO-1 antibody and analyzed via immunoblotting using an anti-p53 or anti-SP1 antibody. The deSUMOylation assay was performed using HA-SUMO-1-vinyl-sulfone (Boston Biochem) as described previously (47).

In vivo ubiquitination assay. In vivo ubiquitination assays to detect SENP1 protein ubiquitination were performed as previously described (18). Briefly, 293T cells were cotransfected with the HA-ubiquitin and FLAG-SENP1 plasmids, and either empty vector or MEL-18-expressing vector, for 48 hours. The cell lysates were immunoprecipitated using an anti-FLAG antibody and analyzed via immunoblotting using an anti-HA or anti-FLAG antibody. To detect endogenous SENP1 protein ubiquitination, MCF-7 cells expressing either MEL-18 or control shRNA were treated with $40 \mu \mathrm{M}$ MG132 for 6 hours. Then, the samples were immunoprecipitated using an anti-SENP1 antibody and subjected to immunoblotting using an anti-Ub antibody.

Orthotopic xenografts and histopathological analysis. Five-week-old female nonobese diabetic/severe combined immunodeficient (NOD/ SCID) mice were purchased from the Korea Research Institute of Bioscience and Biotechnology (Daejeon, Korea). To investigate the effect of MEL-18 on estrogen-independent tumor growth, $4 \times 10^{6} \mathrm{MCF}-7$ or T47D luminal cells expressing either lentiviral MEL-18 or control shRNA were injected into the mammary fat pads of female NOD/SCID mice on the left side (control) and the right side (MEL-18 shRNA) without the implantation of E2 pellets, and tumor formation was monitored. To examine the effect of MEL-18 on antiestrogen treatment, the mice were implanted subcutaneously with or without E2 pellets $(0.72$ $\mathrm{mg} /$ pellet; 60-day release) 1 week before cancer cell implantation. Approximately $4 \times 10^{6}$ control or MEL-18-silenced MCF-7 cells were then injected into the fat pads of the mice as described previously. After 1 week, the experimental animals were subcutaneously injected with or without a tamoxifen pellet (5 mg/pellet; 60 -day release). For the combined treatment of xenografted mice with a PI3K inhibitor and tamoxifen, NOD/SCID mice bearing control or MEL-18-silenced MCF-7 cell-based tumors that were implanted with E2 pellets were subcutaneously injected with tamoxifen as described previously and/ or treated with BKM120 $(30 \mathrm{mg} / \mathrm{kg}$, dissolved in a freshly prepared solution of $10 \%$ NMP and 90\% PEG300) via oral gavage once daily for 2 weeks (6 days on/1 day off, 6IW) and twice weekly for an additional 2 weeks. To evaluate the effect of MEL-18 overexpression on the response of TNBC to tamoxifen in vivo, the NOD/SCID mice were orthotopically xenografted with $1 \times 10^{6}$ MDA-MB-231 cells or $4 \times 10^{6}$ MDA-MB-468 cells expressing either empty vector or lentiviral MEL18 cDNA and were subcutaneously injected with tamoxifen $(5 \mathrm{mg})$ or placebo pellets 1 week after cell implantation. Tumor growth was measured twice weekly for 4-7 weeks. The tumor volume was calculated as $1 / 2 \times$ long diameter $\times$ short diameter ${ }^{2}$.

After the tumors were dissected, IHC analysis was performed using consecutive sections of tumors from 3 independent xenografted mice as described previously $(13,48)$. The results were scored by multiplying the percentage of positive cells by the staining intensity as described in the IHC staining section. A total score of 4 was selected as the threshold value. The results of the Ki- 67 proliferative index and the apoptotic index based on TUNEL staining (Millipore) were quantified by measuring the percentage of positive cells.

Statistics. The significance of the differences between the controls and the experimental groups was determined using a 2-tailed Student's $t$ test. For multiple group comparisons and repeated measures of in vivo data, Welch ANOVA followed by Dunnett's T3 test for unequal variances or repeated-measures ANOVA (RM ANOVA) followed by a post hoc LSD test for equal variances, respectively, were performed. Fisher's exact test was used to examine the correlation of MEL-18 expression with the expression of hormonal receptors and SENP1, and with the clinicopathological variables in the human samples. The Kaplan-Meier curves for OS and DFS analyses were evaluated using the log-rank test. Univariate and multivariate survival analyses using Cox proportional hazard regression models were performed to evaluate independent prognostic factors. These analyses were performed using SPSS software (version 12.0). The difference in the MEL-18 expression levels between the breast cancer subtypes was analyzed via ANOVA with pairwise comparisons. Spearman's rank correlation coefficient was used for the analysis of the correlation between 2 genes. Both analyses were conducted using the R statistical package (http:// www.r-project.org/). The Poisson distribution was calculated to evaluate the significance of the tumor incidence using SAS software. In all cases, $P$ values of $<0.05$ were considered significant.

Study approval. All animal experiments were approved and performed in accordance with the Hanyang University Animal Care and Use Committee.

\section{Acknowledgments}

This work was supported by grants from the National Research Foundation of Korea (NRF) funded by the Korean government (No. 2010-0020879 and 2013R1A1A2012761).

Address correspondence to: Gu Kong, Department of Pathology, College of Medicine, Hanyang University, 17 Haengdang-dong, Seongdong-gu, Seoul, 133-791, South Korea. Phone: 82.2.2290.8251; E-mail: gkong@hanyang.ac.kr. 
1. Ali S, Coombes RC. Estrogen receptor alpha in human breast cancer: occurrence and significance. J Mammary Gland Biol Neoplasia. 2000;5(3):271-281.

2. Giacinti L, Claudio PP, Lopez M, Giordano A. Epigenetic information and estrogen receptor alpha expression in breast cancer. Oncologist . 2006;11(1):1-8.

3. Keen JC, et al. A novel histone deacetylase inhibitor, scriptaid, enhances expression of functional estrogen receptor alpha (ER) in ER negative human breast cancer cells in combination with 5-aza 2'-deoxycytidine. Breast Cancer Res Treat. 2003;81(3):177-186.

4. Yang X, Phillips DL, Ferguson AT, Nelson WG, Herman JG, Davidson NE. Synergistic activation of functional estrogen receptor (ER)- $\alpha$ by DNA methyltransferase and histone deacetylase inhibition in human ER- $\alpha$-negative breast cancer cells. Cancer Res. 2001;61(19):7025-7029.

5. Peddi PF, Ellis MJ, Ma C. Molecular basis of triple negative breast cancer and implications for therapy. Int J Breast Cancer. 2012;2012:217185.

6. Sauvageau M, Sauvageau G. Polycomb group proteins: multi-faceted regulators of somatic stem cells and cancer. Cell Stem Cell. 2010;7(3):299-313.

7. Guo WJ, et al. Mel-18 acts as a tumor suppressor by repressing Bmi-1 expression and down-regulating Akt activity in breast cancer cells. Cancer Res. 2007;67(11):5083-5089.

8. Wang W, et al. The novel tumor-suppressor Mel-18 in prostate cancer: its functional polymorphism, expression and clinical significance. Int $J$ Cancer. 2009;125(12):2836-2843.

9. Riis ML, Luders T, Nesbakken AJ, Vollan HS, Kristensen V, Bukholm IR. Expression of BMI-1 and Mel-18 in breast tissue - a diagnostic marker in patients with breast cancer. $B M C$ cancer. 2010;10:686.

10. Guo BH, et al. Low expression of Mel-18 predicts poor prognosis in patients with breast cancer. Ann Oncol. 2010;21(12):2361-2369.

11. Zhang XW, et al. BMI1 and Mel-18 oppositely regulate carcinogenesis and progression of gastric cancer. Mol Cancer. 2010;9:40.

12. Won HY, et al. Loss of Mel-18 enhances breast cancer stem cell activity and tumorigenicity through activating Notch signaling mediated by the Wnt/TCF pathway. FASEB J. 2012;26(12):5002-5013.

13. Park JH, Lee JY, Shin DH, Jang KS, Kim HJ, Kong G. Loss of Mel-18 induces tumor angiogenesis through enhancing the activity and expression of HIF- $1 \alpha$ mediated by the PTEN/PI3K/Akt pathway. Oncogene. 2011;30(45):4578-4589.

14. Lee JY, et al. Mel-18 negatively regulates INK4a/ ARF-independent cell cycle progression via Akt inactivation in breast cancer. Cancer Res. 2008;68(11):4201-4209.

15. Lee JY, et al. Loss of the polycomb protein Mel-18 enhances the epithelial-mesenchymal transition by ZEB1 and ZEB2 expression through the downregulation of miR-205 in breast cancer. Oncogene. 2014;33(10):1325-1335.
16. Wang H, et al. Role of histone H2A ubiquitination in Polycomb silencing. Nature. 2004;431(7010):873-878.

17. Ohtsubo M, et al. Polycomb-group complex 1 acts as an E3 ubiquitin ligase for Geminin to sustain hematopoietic stem cell activity. Proc Natl Acad Sci U S A. 2008;105(30):10396-10401.

18. Qian T, Lee JY, Park JH, Kim HJ, Kong G. Id1 enhances RING1b E3 ubiquitin ligase activity through the Mel-18/Bmi-1 polycomb group complex. Oncogene. 2010;29(43):5818-5827.

19. Zhang J, Goodson ML, Hong Y, Sarge KD. MEL-18 interacts with HSF2 and the SUMO E2 UBC9 to inhibit HSF2 sumoylation. J Biol Chem. 2008;283(12):7464-7469.

20. Zhang J, Sarge KD. Mel-18 interacts with RanGAP1 and inhibits its sumoylation. Biochem Biophys Res Commun. 2008;375(2):252-255.

21. Richardson AL, et al. X chromosomal abnormalities in basal-like human breast cancer. Cancer Cell. 2006;9(2):121-132.

22. Li Y, et al. Amplification of LAPTM4B and YWHAZ contributes to chemotherapy resistance and recurrence of breast cancer. Nat Med. 2010;16(2):214-218.

23. Shi L, et al. The MicroArray Quality Control (MAQC)-II study of common practices for the development and validation of microarray-based predictive models. Nat Biotechnol. 2010;28(8):827-838.

24. Hatzis C, et al. A genomic predictor of response and survival following taxane-anthracycline chemotherapy for invasive breast cancer. JAMA. 2011;305(18):1873-1881.

25. Chin K, et al. Genomic and transcriptional aberrations linked to breast cancer pathophysiologies. Cancer Cell. 2006;10(6):529-541.

26. Gibson GR, Qian D, Ku JK, Lai LL. Metaplastic breast cancer: clinical features and outcomes. Am Surg. 2005;71(9):725-730.

27. Bos PD, et al. Genes that mediate breast cancer metastasis to the brain. Nature. 2009;459(7249):1005-1009.

28. Gyorffy B, et al. An online survival analysis tool to rapidly assess the effect of 22,277 genes on breast cancer prognosis using microarray data of 1,809 patients. Breast Cancer Res Treat. 2010;123(3):725-731.

29. Kos M, Reid G, Denger S, Gannon F. Minireview: genomic organization of the human ER $\alpha$ gene promoter region. Mol Endocrinol. 2001;15(12):2057-2063.

30. Osborne CK, Schiff R. Mechanisms of endocrine resistance in breast cancer. Annu Rev Med. 2011;62:233-247.

31. Macaluso M, Montanari M, Noto PB, Gregorio V, Bronner C, Giordano A. Epigenetic modulation of estrogen receptor- $\alpha$ by $\mathrm{pRb}$ family proteins: a novel mechanism in breast cancer. Cancer Res. 2007;67(16):7731-7737.

32. Shirley SH, et al. Transcriptional regulation of estrogen receptor-alpha by p53 in human breast cancer cells. Cancer Res. 2009;69(8):3405-3414.

33. Liu B, Shuai K. Regulation of the sumoylation system in gene expression. Curr Opin Cell Biol.
2008;20(3):288-293.

34. Lin JY, Ohshima T, Shimotohno K. Association of Ubc9, an E2 ligase for SUMO conjugation, with $\mathrm{p} 53$ is regulated by phosphorylation of $\mathrm{p} 53$. FEBS Lett. 2004;573(1-3):15-18.

35. Spengler ML, Kennett SB, Moorefield KS, Simmons SO, Brattain MG, Horowitz JM. Sumoylation of internally initiated $\mathrm{Sp} 3$ isoforms regulates transcriptional repression via a Trichostatin A-insensitive mechanism. Cell Signal. 2005;17(2):153-166.

36. Grinkevich VV, et al. Ablation of key oncogenic pathways by RITA-reactivated p53 is required for efficient apoptosis. Cancer Cell. 2009;15(5):441-453.

37. Li H, Zhang Y, Strose A, Tedesco D, Gurova $\mathrm{K}$, Selivanova G. Integrated high-throughput analysis identifies Sp1 as a crucial determinant of p53-mediated apoptosis. Cell Death Differ. 2014;21(9):1493-1502.

38. Miller TW, et al. Hyperactivation of phosphatidylinositol-3 kinase promotes escape from hormone dependence in estrogen receptor-positive human breast cancer. J Clin Invest. 2010;120(7):2406-2413.

39. Shah SP, et al. The clonal and mutational evolution spectrum of primary triple-negative breast cancers. Nature. 2012;486(7403):395-399.

40. Iqbal J, Thike AA, Cheok PY, Tse GM, Tan PH. Insulin growth factor receptor-1 expression and loss of PTEN protein predict early recurrence in triple-negative breast cancer. Histopathology. 2012;61(4):652-659.

41. Turner N, et al. Targeting triple negative breast cancer: is p53 the answer? Cancer Treat Rev. 2013;39(5):541-550.

42. Wu SY, Chiang CM. Crosstalk between sumoylation and acetylation regulates p53-dependent chromatin transcription and DNA binding. EMBO J. 2009;28(9):1246-1259.

43. Schmidt D, Muller S. Members of the PIAS family act as SUMO ligases for c-Jun and p53 and repress 553 activity. Proc Natl Acad Sci US A. 2002;99(5):2872-2877.

44. Ahn BY, et al. Genetic screen identifies insulinlike growth factor binding protein 5 as a modulator of tamoxifen resistance in breast cancer. Cancer Res. 2010;70(8):3013-3019.

45. Parra-Palau JL, et al. A major role of p95/611CTF, a carboxy-terminal fragment of HER2, in the down-modulation of the estrogen receptor in HER2-positive breast cancers. Cancer Res. 2010;70(21):8537-8546.

46. Neve RM, et al. A collection of breast cancer cell lines for the study of functionally distinct cancer subtypes. Cancer Cell. 2006;10(6):515-527.

47. Kolli N, et al. Distribution and paralogue specificity of mammalian deSUMOylating enzymes. Biochem J. 2010;430(2):335-344.

48. Shin DH, Jang SH, Kang BC, Kim HJ, Oh SH, Kong G. Constitutive overexpression of Id-1 in mammary glands of transgenic mice results in precocious and increased formation of terminal end buds, enhanced alveologenesis, delayed involution. JCell Physiol. 2011;226(5):1340-1352. 